\title{
From Heisenberg-Euler Lagrangian to the discovery of Chromomagnetic Gluon Condensation
}

\author{
George Savvidy ${ }^{\mathrm{a}}$ (B) \\ Institute of Nuclear and Particle Physics, Demokritos National Research Center, Agia Paraskevi, 15310 Athens, Greece
}

Received: 20 October 2019 / Accepted: 4 February 2020 / Published online: 21 February 2020

(C) The Author(s) 2020

\begin{abstract}
I reexamine the phenomena of the chromomagnetic gluon condensation in Yang-Mills theory. The extension of the Heisenberg-Euler Lagrangian to the Yang-Mills theory allows to calculate the effective action, the energymomentum tensor and demonstrate that the energy density curve crosses the zero energy level of the perturbative vacuum state at nonzero angle and continuously enters to the negative energy density region. At the crossing point and further down the effective coupling constant is small and demonstrate that the true vacuum state of the Yang-Mills theory is below the perturbative vacuum state and is described by the nonzero chromomagnetic gluon condensate. The renormalisation group analyses allows to express the energy momentum tensor, its trace and the vacuum magnetic permeabilities in QED and QCD in terms of effective coupling constant and Callan-Symanzik beta function. In the vacuum the energymomentum tensor is proportional to the space-time metric, and it induces a negative contribution to the effective cosmological constant.
\end{abstract}

\section{Introduction}

In this article we shall analyse the effective action in QED and QCD by using the perturbative loop expansion and renormalisation group equations and discuss the physical consequences which can be derived from their explicit expressions. We shall reexamine the phenomena of the chromomagnetic gluon condensation in Yang-Mills (YM) theory and will present the derivation of the new results. The Heisenberg-

Based on lectures at the Leipzig University in occasion of the 80 Years of Heisenberg-Euler Lagrangian 1936-2016, ITP, Leipzig, November 21, 2016 and 40 Years of Discovery of the Chromomagnetic Gluon Condensation 1977-2017 at the Ludwig-Maximilian University München, Arnold Sommerfeld Colloquium at Center for Theoretical Physics, April 18, 2018.

a e-mail: savvidy@inp.demokritos.gr (corresponding author)
Euler Lagrangian in QED [1-6] is a sum of the one loop diagrams with a vacuum electron-positron pair circulating in the loop and the gluons and quarks in case of QCD [7-16]. The effective action $\Gamma[A]$ has the following representation:

$$
\begin{aligned}
\Gamma= & \int \mathcal{L} d x=\sum_{n} \int d x_{1} \ldots d x_{n} \Gamma_{\mu_{1} \ldots \mu_{n}}^{(n) a_{1} \ldots a_{n}}\left(x_{1}, \ldots, x_{n}\right) \\
& \times A_{\mu_{1}}^{a_{1}}\left(x_{1}\right) \ldots A_{\mu_{n}}^{a_{n}}\left(x_{n}\right)=S+W^{(1)}+W^{(2)}+\cdots,
\end{aligned}
$$

where $\mathcal{L}$ is the effective Lagrangian, $\Gamma^{(n)}$ is a one-particle irreducible (1PI) vertex function, $A_{\mu}^{a}(x) \equiv\left\langle 0\left|A_{\mu}^{a}(x)\right| 0\right\rangle$ is the vacuum expectation value of the field operator and $W^{(n)}, n=1,2, \ldots$ represent the terms of the loop expansion.

We shall consider the limit of massless electrons and quarks and demonstrate that the proper time integral in the Heisenberg-Euler Lagrangian can be calculated explicitly by using covariant renormalisation condition $[12,15,16]$

$$
\left.\frac{\partial \mathcal{L}}{\partial \mathcal{F}}\right|_{t=\frac{1}{2} \ln \left(\frac{2 e^{2}|\mathcal{F}|}{\mu^{4}}\right)=\mathcal{G}=0}=-1,
$$

where $\mathcal{F}=\frac{1}{4} G_{\mu \nu}^{a} G_{\mu \nu}^{a}$ is the Lorentz and gauge invariant form of the YM field strength tensor $G_{\mu \nu}^{a}$ and $\mu^{2}$ is the renormalisation scale parameter. In the massless limit the QED effective Lagrangian has the exact logarithmic dependence as a function of the invariant $\mathcal{F}$ (see Fig. 1):

$$
\begin{aligned}
\mathcal{L}_{e} & =-\mathcal{F}+\frac{e^{2} \mathcal{F}}{24 \pi^{2}}\left[\ln \left(\frac{2 e^{2} \mathcal{F}}{\mu^{4}}\right)-1\right], \\
\mathcal{F} & =\frac{\overrightarrow{\mathcal{H}}^{2}-\overrightarrow{\mathcal{E}}^{2}}{2}, \quad \mathcal{G}=\overrightarrow{\mathcal{E}} \overrightarrow{\mathcal{H}}=0,
\end{aligned}
$$

where $\overrightarrow{\mathcal{H}}$ and $\overrightarrow{\mathcal{E}}$ are magnetic and electric fields. This expression should be compared with the one-loop effective Lagrangian in pure $\mathrm{SU}(\mathrm{N})$ gauge field theory, which has the form [12,15] (see Fig. 2): 


$$
\begin{gathered}
\mathcal{L}_{g}=-\mathcal{F}-\frac{11 N}{96 \pi^{2}} g^{2} \mathcal{F}\left(\ln \frac{2 g^{2} \mathcal{F}}{\mu^{4}}-1\right), \\
\mathcal{F}=\frac{\overrightarrow{\mathcal{H}}_{a}^{2}-\overrightarrow{\mathcal{E}}_{a}^{2}}{2}>0, \quad \mathcal{G}=\overrightarrow{\mathcal{E}}_{a} \overrightarrow{\mathcal{H}}_{a}=0 .
\end{gathered}
$$

From (1.3) it follows that the corresponding quark contribution considered in the chiral limit is

$$
\mathcal{L}_{q}=-\mathcal{F}+\frac{N_{f}}{48 \pi^{2}} g^{2} \mathcal{F}\left[\ln \left(\frac{2 g^{2} \mathcal{F}}{\mu^{4}}\right)-1\right],
$$

where $N_{f}$ is the number of quark flavours.

The effective Lagrangian technique allows to calculate the magnetic induction $\overrightarrow{\mathcal{B}}$ of the vacuum defined through the derivative of the effective Lagrangian [12]:

$\overrightarrow{\mathcal{B}}_{a}=-\frac{\partial \mathcal{L}}{\partial \overrightarrow{\mathcal{H}}_{a}}=\mu_{v a c} \overrightarrow{\mathcal{H}}_{a}$

From (1.3), (1.4) and (1.5) it follows that in QED the vacuum responds to the background magnetic field as diamagnet and in QCD as paramagnet with the magnetic permeabilities of the following form [12]:

\section{$\mu_{Q E D}$}

$$
=1-\frac{e^{2}}{24 \pi^{2}} \log \left(\frac{e^{2} \overrightarrow{\mathcal{H}}^{2}}{\mu^{4}}\right)<1, \quad \text { diamagnetic, }
$$

$\mu_{Q C D}$

$$
\begin{aligned}
& =1+\frac{g^{2}}{96 \pi^{2}}\left(11 N-2 N_{f}\right) \log \frac{g^{2} \overrightarrow{\mathcal{H}}_{a}^{2}}{\mu^{4}}>1, \\
& \text { paramagnetic, } \quad N>\frac{2}{11} N_{f} .
\end{aligned}
$$

The diamagnetism of the QED vacuum (1.7) means that it repels the magnetic fields by forming induced magnetic field in the direction opposite to that of the applied magnetic field. This phenomenon is similar to the Landau orbital diamagnetism of free electron gas when the counteracting field is formed when the electron trajectories are curved due to the Lorentz force [87]. The paramagnetism of the QCD vacuum (1.8) means that it amplifies the applied chromomagnetic field by generating induced chromomagnetic field in the direction of the applied field. In QCD the large polarisation of the gluon spins is responsible for the amplification of the background field. This phenomenon is similar to the Pauli paramagnetism, an effect associated with the polarisation of the electron spins [86].

The effective Lagrangian approach allows to calculate the quantum-mechanical corrections to the energy momentum tensor by using the formula derived by Schwinger in [5]:

$$
\begin{aligned}
T_{\mu \nu}= & \left(F_{\mu \lambda} F_{\nu \lambda}-g_{\mu \nu} \frac{1}{4} F_{\lambda \rho}^{2}\right) \frac{\partial \mathcal{L}}{\partial \mathcal{F}} \\
& -g_{\mu \nu}\left(\mathcal{L}-\mathcal{F} \frac{\partial \mathcal{L}}{\partial \mathcal{F}}-\mathcal{G} \frac{\partial \mathcal{L}}{\partial \mathcal{G}}\right) .
\end{aligned}
$$

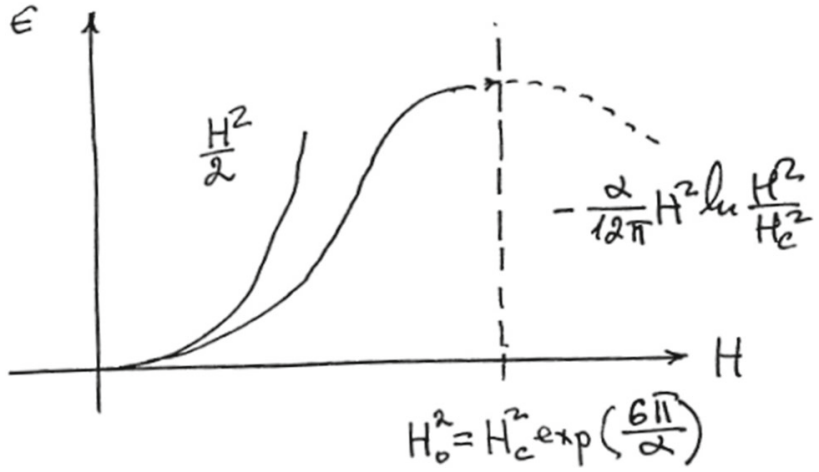

Fig. 1 The graph shows the qualitative behaviour of the QED vacuum energy density $\epsilon\left(\overrightarrow{\mathcal{H}}^{2}\right)(1.3),(2.19)$ and of the effective coupling constant $\bar{e}^{2}\left(\mathcal{\mathcal { H }}^{2}\right)(4.3)$ as the functions of the background magnetic field. The effective coupling constant is singular at $\overrightarrow{\mathcal{H}}_{0}{ }^{2}$, the "Moscow zero" [90, 91]

In case of the Heisenberg-Euler effective Lagrangian Schwinger presented the expression for the $T_{\mu \nu}$ in the fine structure constant $\alpha=e^{2} / 4 \pi$ expansion:

$$
\begin{aligned}
T_{\mu \nu}= & T_{\mu \nu}^{M}\left(1-\frac{16}{45 m^{4}} \alpha^{2} \mathcal{F}\right) \\
& +g_{\mu \nu} \frac{2}{45 m^{4}} \alpha^{2}\left(4 \mathcal{F}^{2}+7 \mathcal{G}^{2}\right)+\cdots
\end{aligned}
$$

with its nonzero trace

$T=T_{\mu \mu}=\frac{8}{45 m^{4}} \alpha^{2}\left(4 \mathcal{F}^{2}+7 \mathcal{G}^{2}\right)+\cdots$

In massless QED using the one-loop expression (1.3) for $T_{\mu \nu}$ one can get

$T_{\mu \nu}=T_{\mu \nu}^{M}\left[1-\frac{e^{2}}{24 \pi^{2}} \ln \frac{2 e^{2} \mathcal{F}}{\mu^{4}}\right]+g_{\mu \nu} \frac{e^{2}}{24 \pi^{2}} \mathcal{F}, \quad \mathcal{G}=0$.

The $T_{\mu \nu}$ becomes proportional to the space-time metric tensor $g_{\mu \nu}$ at the extreme magnetic field $H_{0}^{2}=H_{c}^{2} \exp (6 \pi / \alpha)$ and therefore induces a positive effective cosmological constant (see Fig. 1).

To calculate the energy momentum tensor $T_{\mu \nu}$ in pure $S U(N)$ YM theory one should use the expression (1.4) and in the case of QCD, in the limit of chiral fermions, one should also add the quark contribution (1.5) by using the substitution $11 N \rightarrow b=11 N-2 N_{f}$ :

$T_{\mu \nu}=T_{\mu \nu}^{Y M}\left[1+\frac{b g^{2}}{96 \pi^{2}} \ln \frac{2 g^{2} \mathcal{F}}{\mu^{4}}\right]-g_{\mu \nu} \frac{b g^{2}}{96 \pi^{2}} \mathcal{F}, \quad \mathcal{G}=0$.

The vacuum energy density $T_{00} \equiv \epsilon(\mathcal{F})$ has therefore the following form [15]:

$\epsilon(\mathcal{F})=\mathcal{F}+\frac{b g^{2}}{96 \pi^{2}} \mathcal{F}\left(\ln \frac{2 g^{2} \mathcal{F}}{\mu^{4}}-1\right)$ 
The energy density has its new minimum outside of the perturbative vacuum state $\left\langle G_{\mu \nu}^{2}\right\rangle=0$, at the Lorentz and renormalisation group invariant field strength [15]

$$
\left\langle 2 g^{2} \mathcal{F}\right\rangle_{v a c}=\mu^{4} \exp \left(-\frac{96 \pi^{2}}{b g^{2}(\mu)}\right)=\Lambda_{Q C D}^{4},
$$

where $b=11 N-2 N_{f}$ and characterises the dynamical breaking of scaling invariance in YM theory ${ }^{1}$ :

$T_{\mu \mu}=-\frac{b}{48 \pi^{2}}\left\langle 2 g^{2} \mathcal{F}\right\rangle_{v a c}$

Substituting the vacuum field intensity (1.15) into the expression for the energy momentum tensor (1.13) one can get that in the vacuum the tensor $T_{\mu \nu}$ is proportional to the space-time metric $g_{\mu \nu}$ :

$\left\langle T_{\mu \nu}\right\rangle_{v a c}=-g_{\mu \nu} \frac{b}{96 \pi^{2}}\left\langle g^{2} \mathcal{F}\right\rangle_{v a c}$.

In this form the energy momentum tensor represents the relativistically invariant equation of state $\epsilon_{v a c}=-P_{v a c}$, which uniquely characterises the vacuum [17-20] with its negative energy density $\epsilon_{v a c}$. The vacuum energy momentum tensor (1.16) generates the effective cosmological constant $\Lambda_{e f f}$

$$
\begin{aligned}
R_{\mu \nu}-\frac{1}{2} g_{\mu \nu} R & =g_{\mu \nu} \Lambda_{e f f}+\frac{8 \pi G}{c^{4}} T_{\mu \nu} \\
& =\frac{8 \pi G}{c^{4}}\left(\left\langle T_{\mu \nu}\right\rangle_{v a c}+T_{\mu \nu}\right)
\end{aligned}
$$

of the form:

$\epsilon_{v a c}=\frac{c^{4} \Lambda_{e f f}}{8 \pi G}=-\frac{b}{96 \pi^{2}}\left\langle g^{2} \mathcal{F}\right\rangle_{v a c}=-\frac{b}{192 \pi^{2}} \Lambda_{Q C D}^{4}$,

where the chromomagnetic condensate (1.15) is $<2 g^{2} \mathcal{F}$ $>_{v a c}=\Lambda_{Q C D}^{4}$. The magnetic permeability (1.8) in the vacuum state (1.15) is equal to zero:

$\mu_{v a c}^{Q C D}=1+\frac{b g^{2}}{96 \pi^{2}} \log \frac{\left\langle 2 g^{2} \mathcal{F}\right\rangle_{v a c}}{\mu^{4}}=0$.

It is useful to derive the expression of the effective Lagrangian by using the renormalisation group equation $[15,16]$. The solution of the renormalisation group equation in terms of effective coupling constant $\bar{g}(g, t)$, with the boundary condition $\bar{g}(g, 0)=g$, has the following form $[15,16]$ :

$\frac{\partial \mathcal{L}}{\partial \mathcal{F}}=-\frac{g^{2}}{\bar{g}^{2}(t)}, \quad \frac{d \bar{g}}{d t}=\beta(\bar{g}), \quad t=\frac{1}{2} \ln \left(2 g^{2} \mathcal{F} / \mu^{4}\right)$.

The derivative (1.19) of the effective Lagrangian has transparent expression in terms of the effective coupling constant

1 The $\Lambda_{Q C D}$ is defined here through the covariant subtraction scheme (1.2). The relation with other renormalisation schemes can be found in [37]. and allows to obtain the effective Lagrangian by integration over $\mathcal{F}$ in all order of the perturbative expansion:

$\mathcal{L}(\mathcal{F})=-\mu^{4} \int \frac{e^{2 t}}{\bar{g}^{2}(t)} d t, \quad t=\frac{1}{2} \ln \left(2 g^{2} \mathcal{F} / \mu^{4}\right)$,

and find out the expressions for the physical quantities beyond the one-loop approximation. One can calculate different observables of physical interest that will include the effective energy momentum tensor, vacuum energy density, the magnetic permeability, the effective coupling constants and their behaviour as a function of the external fields. In particular, the energy momentum tensor (1.9) will take the following form:

$$
\begin{aligned}
T_{\mu \nu}= & -\left(G_{\mu \lambda} G_{\nu \lambda}-g_{\mu \nu} \frac{1}{4} G_{\lambda \rho}^{2}\right) \frac{g^{2}}{\bar{g}^{2}(t)} \\
& +g_{\mu \nu}\left(\int \frac{e^{2 t}}{\bar{g}^{2}(t)} d t-\frac{1}{2} \frac{e^{2 t}}{\bar{g}^{2}(t)}\right) \mu^{4} .
\end{aligned}
$$

And the vacuum energy density can be expressed in terms of the trace $T_{\mu \mu}$ :

$\varepsilon=T_{00}=\frac{\overrightarrow{\mathcal{H}}_{a}^{2}}{2} \frac{g^{2}}{\bar{g}^{2}(t)}+\frac{1}{4} T_{\mu \mu}, \quad \mathcal{G}=0$,

where the trace of the energy momentum tensor $T_{\mu \mu}$ is given by the following expression:

$T_{\mu \mu}=4 \mu^{4} \int \frac{e^{2 t} \beta(\bar{g}(t))}{\bar{g}(t)^{3}} d t, \quad t=\frac{1}{2} \ln \left(2 g^{2} \mathcal{F} / \mu^{4}\right)$.

The last formula provides all-loop expression for the conformal anomaly in gauge field theories. ${ }^{2}$ As far as the beta function $\bar{\beta}(\mathrm{g})$ has no zeros, is negative analytical function of the coupling constant and

$\int_{g}^{\infty} \frac{d g}{\beta(g)}<\infty$

the minimum of the energy density curve is defined by the extremum, where the derivative (1.19) vanishes. It follows that the value of the chromomagnetic condensate is [15]

$$
\left\langle 2 g^{2} \mathcal{F}\right\rangle_{\text {vac }}=\mu^{4} \exp \left(2 \int_{g(\mu)}^{\infty} \frac{d g}{\beta(g)}\right) .
$$

Considering the value of the field strength tensor $\mathcal{F}_{0}$ at which the vacuum energy density (1.14) vanishes $\epsilon\left(\mathcal{F}_{0}\right)=0$, the point $\mathcal{F}_{0}$ shown on Fig. 2, one can observe that the effective coupling constant (1.19) at this field strength has the value $\frac{96 \pi^{2}}{11 N-2 N_{f}}$ and tends to zero as $N \rightarrow \infty$. The energy density curve $\epsilon(\mathcal{F})$ (1.14) intersect the horizontal zero energy line at the nonzero angle $\theta>0$ (see

\footnotetext{
$\overline{2}$ If one considers the approximation in which $\bar{g}(t)$ is field independent $\bar{g}(t) \equiv g$ then (1.23) will reduce to the one given in literature [56-60].
} 


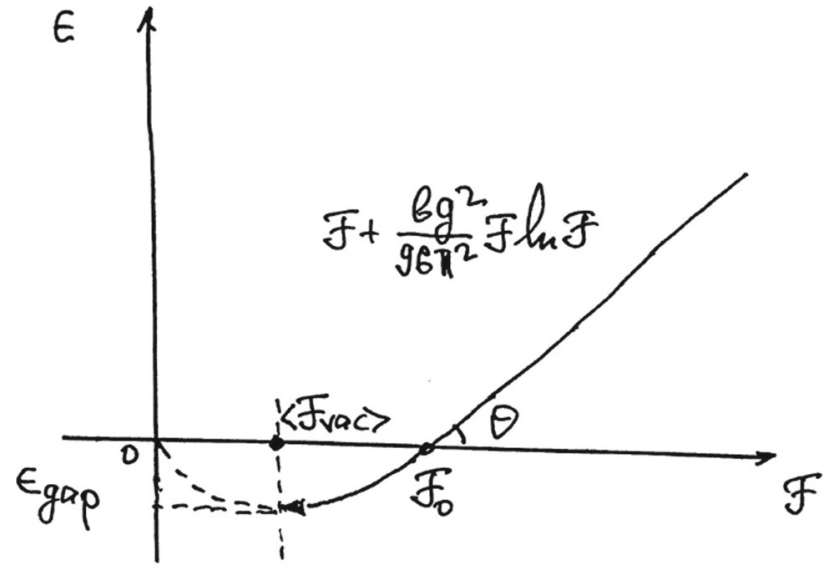

Fig. 2 The graph shows the qualitative behaviour of the vacuum energy density $\epsilon(\mathcal{F})(1.14),(4.16)$. At the intersection point $\mathcal{F}_{0}(6.17)$ the effective coupling constant is small (6.18) and the intersection angle $\theta$ is strictly positive (6.19). The energy density curve can be continuously extended from the point $\mathcal{F}_{0}$ deep into the negative energy density region arbitrary close to the value of the vacuum condensate $\langle\mathcal{F}\rangle_{\text {vac }}$ by considering a larger values of $N$ and keeping the t'Hooft coupling constant $g^{2} N$ fixed (6.20), (6.21). This demonstrate that there is a nonzero energy gap $\epsilon_{\text {gap }}>0$ between perturbative and true vacuum states. The vacuum is characterised by the nonzero value of the chromomagnetic field strength tensor (1.15), (6.8) and the energy density gap $\epsilon_{\text {gap }}=\left|\epsilon_{\text {vac }}\right|$ (1.17) $[15,37]$

(6.19) and Fig. 2). The energy density curve can be continuously extended from the point $\mathcal{F}_{0}$ deep into the negative energy density region arbitrary close to the value of the vacuum condensate $\langle\mathcal{F}\rangle_{\text {vac }}$ by considering a larger values of $N$ and keeping the t'Hooft coupling constant $g^{2} N$ small and fixed. This demonstrates that the true vacuum of the Yang-Mills theory is below the perturbative vacuum, the energy density curve $\epsilon(\mathcal{F})$ is approaching the point of zero energy density from below and that there exists a nonzero energy gap between perturbative and true vacuum states.

The article is organised as follows. In the Sect. 2 we shall use gauge and renormalisation group invariant scheme (1.2) $[12,15]$ to renormalise the massless HeisenbergEuler Lagrangian and derive the exact one-loop expression for the effective Lagrangian in QED (1.3). In the Sect. 3 we shall use the renormalisation group equations for the effective Lagrangian to derive all loops results for the vacuum energy density and the traces of the energy momentum tensor. In Sects. 4 and 5 the analyses will be extended to the Yang-Mills theory and the phenomena of the chromomagnetic gluon condensation will be reexamined. We shall present the absence of the imaginary part in the YM effective Lagrangian in chromomagnetic field and the stability of the chromomagnetic gluon condensate by Niels Bohr theory group and by Kurt Flory.

\section{Heisenberg-Euler effective Lagrangian in massless limit}

The effective action $\Gamma$ and the effective Lagrangian $\mathcal{L}$ in gauge field theories can be represented as a sum of the oneparticle irreducible loop diagrams $W^{(n)}, n=1,2 \ldots$ :

$\Gamma=\int \mathcal{L} d^{4} x=S+W^{(1)}+W^{(2)}+\cdots$,

where $S$ is the Yang-Mills or Maxwell action:

$S=-\frac{1}{4} \int G_{\mu \nu}^{2} d^{4} x$

We shall analyse the behaviour of the effective actions in both theories and shall consider first the Quantum Electrodynamic in massless limit (see, in particular, [83]). The HeisenbergEuler Lagrangian in QED [1-6,82] is a sum of the one-loop diagrams with a vacuum electron-positron pair running in the loop:

$W^{(1)}=\int \mathcal{L}^{(1)}(x) d^{4} x$,

and can be expressed through the functional determinant of the Dirac operator [5]:

$$
\begin{aligned}
W^{(1)} & =-i \operatorname{Tr} \ln (\gamma \Pi+m)=i \int_{0}^{\infty} \frac{d s}{s} \operatorname{Tr} e^{-i(\gamma \Pi+m) s} \\
& =\frac{i}{2} \int_{0}^{\infty} \frac{d s}{s} \operatorname{Tr} e^{-i\left(m^{2}-(\gamma \Pi)^{2}\right) s}
\end{aligned}
$$

where $\Pi_{\mu}=-i \partial_{\mu}-e A_{\mu}$. The general expression for the one-loop effective Lagrangian $\mathcal{L}^{(1)}$ has therefore the following form:

$\mathcal{L}^{(1)}(x)=\frac{i}{2} \int_{0}^{\infty} \frac{d s}{s} e^{-i m^{2} s} \operatorname{tr}(x|U(s)| x)$,

where

$U(s)=e^{-i H s}, \quad H=-(\gamma \Pi)^{2}=\Pi_{\mu}^{2}-\frac{1}{2} e \sigma_{\mu \nu} F_{\mu \nu}$.

In the case of the constant electromagnetic field strength tensor $F_{\mu \nu}$ the matrix element of the operator $(x|U(s)| x)$ can be calculated exactly and has the following form [5]:

$(x|U(s)| x)=-\frac{i}{(4 \pi s)^{2}} e^{-L(s)} e^{\frac{i}{2} e \sigma F s}$,

where

$L(s)=\frac{1}{2} \operatorname{tr} \ln \left[(e F s)^{-1} \sinh (e F s)\right]$.

The Lagrangian will take the form

$\mathcal{L}^{(1)}=\frac{1}{32 \pi^{2}} \int_{0}^{\infty} \frac{d s}{s^{3}} e^{-i m^{2} s} e^{-L(s)} \operatorname{tr} e^{\frac{i}{2} e \sigma F s}$. 
By the rotation of the integration contour in complex plane (5) as $s \rightarrow-i s$ one can get

$\mathcal{L}^{(1)}=-\frac{1}{32 \pi^{2}} \int_{0}^{\infty} \frac{d s}{s^{3}} e^{-m^{2} s} e^{-l(s)} \operatorname{tr} e^{\frac{1}{2} e \sigma F s}$,

where

$l(s)=\frac{1}{2} \operatorname{trln}\left[(e F s)^{-1} \sin (e F s)\right]$.

The traces in (2.6) can be evaluated by using the eigenvalues of the field strength tensor matrix $F_{\mu \nu} \psi_{\nu}=F \psi_{\mu}$. The characteristic equation is

$F^{4}+2 \mathcal{F} F^{2}-\mathcal{G}^{2}=0$,

where

$\mathcal{F}=\frac{1}{4} F_{\mu \nu}^{2}=\frac{1}{2}\left(\overrightarrow{\mathcal{H}}^{2}-\overrightarrow{\mathcal{E}}^{2}\right), \quad \mathcal{G}=\frac{1}{4} F_{\mu \nu} F_{\mu \nu}^{*}=\overrightarrow{\mathcal{E}} \overrightarrow{\mathcal{H}}$,

and has the solutions

$F_{1}^{2}=-\mathcal{F}-\left(\mathcal{F}^{2}+\mathcal{G}^{2}\right)^{1 / 2}, \quad F_{2}^{2}=-\mathcal{F}+\left(\mathcal{F}^{2}+\mathcal{G}^{2}\right)^{1 / 2}$.

Thus

$$
\begin{aligned}
e^{-l(s)}= & \frac{e F_{1} s e F_{2} s}{\sin \left(e F_{1} s\right) \sin \left(e F_{2} s\right)}, \\
& \operatorname{tr} e^{\frac{1}{2} e \sigma F s}=4 \cos \left(e F_{1} s\right) \cos \left(e F_{2} s\right) .
\end{aligned}
$$

The Lagrangian (2.6) will take the following form [4, 5]:

$$
\begin{aligned}
\mathcal{L}^{(1)}= & -\frac{1}{8 \pi^{2}} \int_{0}^{\infty} \frac{d s}{s^{3}} \\
& e^{-m^{2} s} \frac{\left(e F_{1} s\right) \cos \left(e F_{1} s\right)\left(e F_{2} s\right) \cos \left(e F_{2} s\right)}{\sin \left(e F_{1} s\right) \sin \left(e F_{2} s\right)},
\end{aligned}
$$

and with real eigenvalues $f_{1}=-i F_{1}, f_{2}=F_{2}$ one can get

$$
\begin{aligned}
\mathcal{L}^{(1)}= & -\frac{1}{8 \pi^{2}} \int_{0}^{\infty} \frac{d s}{s^{3}} \\
& e^{-m^{2} s} \frac{\left(e f_{1} s\right) \cosh \left(e f_{1} s\right)\left(e f_{2} s\right) \cos \left(e f_{2} s\right)}{\sinh \left(e f_{1} s\right)}, \sin \left(e f_{2} s\right)
\end{aligned}
$$

where

$$
\begin{aligned}
& f_{1}=\left(\left(\mathcal{F}^{2}+\mathcal{G}^{2}\right)^{1 / 2}+\mathcal{F}\right)^{1 / 2}, \\
& f_{2}=\left(\left(\mathcal{F}^{2}+\mathcal{G}^{2}\right)^{1 / 2}-\mathcal{F}\right)^{1 / 2} .
\end{aligned}
$$

In pure magnetic field configurations $\mathcal{G}=0, \mathcal{F}>$ 0 one can get $f_{1}=(2 \mathcal{F})^{1 / 2}, f_{2}=0$ and in pure electric case $\mathcal{G}=0, \mathcal{F}<0$, thus $f_{1}=0, f_{2}=$ $(-2 \mathcal{F})^{1 / 2}$ :

$\mathcal{G}=0, \mathcal{F}>0 \quad f_{1}=(2 \mathcal{F})^{1 / 2}, f_{2}=0 \quad$ pure magnetic $\mathcal{G}=0, \mathcal{F}<0 \quad f_{1}=0, f_{2}=(-2 \mathcal{F})^{1 / 2} \quad$ pure electric.
We shall consider the QED in the massless limit and impose the following renormalisation condition on the effective Lagrangian introduced in $[15,16]^{3}$ :

$$
\left.\frac{\partial \mathcal{L}}{\partial \mathcal{F}}\right|_{t=\frac{1}{2} \ln \left(\frac{2 e^{2}|\mathcal{F}|}{\mu^{4}}\right)=\mathcal{G}=0}=-1
$$

where $\mu^{2}$ is the renormalisation scale parameter. This condition defines the renormalisation of the effective Lagrangian in a covariant gauge $\mathcal{L}_{r}=\mathcal{L}_{u n}-Z \mathcal{F}$. In the case of pure magnetic field (2.11) the Lagrangian $\mathcal{L}^{(1)}$ has the following form:

$\mathcal{L}^{(1)}=-\frac{1}{8 \pi^{2}} \int_{0}^{\infty} \frac{d s}{s^{3}} \frac{\left(e f_{1} s\right) \cosh \left(e f_{1} s\right)}{\sinh \left(e f_{1} s\right)}$,

and diverges at the boundaries of the proper time integration region. With the use of the renormalisation condition (2.12) one can handle both divergences $[15,16]$. This leads to the following renormalisation of the Heisenberg-Euler Lagrangian in the massless limit:

$$
\begin{aligned}
\mathcal{L}^{(1)}= & -\frac{\mu^{4}}{8 \pi^{2}} \int_{0}^{\infty} \frac{d s}{s^{3}} \\
& \times\left(\frac{a s \cosh (a s)}{\sinh (a s)}-1-\frac{a^{2} s}{2}\left(\frac{\cosh s}{\sinh s}-\frac{s}{\sinh ^{2} s}\right)\right),
\end{aligned}
$$

where

$a^{2}=2 e^{2} \mathcal{F} / \mu^{4}=e^{2} \overrightarrow{\mathcal{H}}^{2} / \mu^{4}, \quad \mathcal{G}=0$.

One can get convinced that this expression is well defined in both limits, in the ultraviolet $s \rightarrow 0$ and in the infrared $s \rightarrow \infty$ regions. One can calculate this integral exactly. The integrals appearing in this expression can be expressed in terms of the Riemann zeta function and its extension (see the Appendix for details). The Lagrangian (2.13) will take the following form:

$$
\begin{aligned}
\mathcal{L}_{k}^{(1)}= & -\frac{\mu^{4}}{8 \pi^{2}} \int_{0}^{\infty} d s \\
& \times\left(\frac{a s^{k-1} \cosh (a s)}{\sinh (a s)}-\frac{a^{2} s^{k-1} \cosh s}{2 \sinh s}+\frac{a^{2} s^{k}}{2 \sinh ^{2} s}\right) \\
= & -\frac{\mu^{4}}{8 \pi^{2}}\left[2 a^{1-k}-a^{2}+a^{2} k\right] \frac{\Gamma(k) \zeta(k)}{2^{k}},
\end{aligned}
$$

and in the limit $k \rightarrow-1$ we shall get

$$
\begin{aligned}
\mathcal{L}^{(1)} & =\frac{\mu^{4} a^{2}}{4 \pi^{2}}\left[\ln a-\frac{1}{2}\right] \lim _{k \rightarrow-1} \frac{(k+1) \Gamma(k) \zeta(k)}{2^{k}} \\
& =\frac{\mu^{4} a^{2}}{24 \pi^{2}}\left[\ln a-\frac{1}{2}\right],
\end{aligned}
$$

3 This renormalisation scheme is alternative to the standard MS and other schemes, see, in particular [37]. 
where we used the identity [73] $\lim _{k \rightarrow-1}(k+1) \Gamma(k) \zeta(k)=$ $\lim _{k \rightarrow-1} \frac{2^{k-1} \pi^{k} \zeta(1-k)}{\cos \left(\frac{\pi k}{2}\right)}=\frac{1}{6}$. Thus, in terms of Lorentz and gauge invariant $\mathcal{F}=\frac{1}{4} F_{\mu \nu}^{2}$, the exact expression of the oneloop Lagrangian in massless QED is:

$\mathcal{L}_{e l}^{(1)}=\frac{e^{2} \mathcal{F}}{24 \pi^{2}}\left[\ln \left(\frac{2 e^{2} \mathcal{F}}{\mu^{4}}\right)-1\right]$,

where $2 \mathcal{F}=\overrightarrow{\mathcal{H}}^{2}-\overrightarrow{\mathcal{E}}^{2}>0, \mathcal{G}=\overrightarrow{\mathcal{E}} \overrightarrow{\mathcal{H}}=0$ and the effective Lagrangian will take the following form:

$\mathcal{L}=-\mathcal{F}+\frac{e^{2} \mathcal{F}}{24 \pi^{2}}\left[\ln \left(\frac{2 e^{2} \mathcal{F}}{\mu^{4}}\right)-1\right]$.

As it follows from this expression, the QED vacuum responds to the background magnetic field by inducing a vacuum current of the electron-positron pairs, which attenuates the magnetic field imposed on the vacuum. The magnetic induction $\overrightarrow{\mathcal{B}}$ of the QED vacuum is [12]:

$\overrightarrow{\mathcal{B}}=-\frac{\partial \mathcal{L}}{\partial \overrightarrow{\mathcal{H}}}=\overrightarrow{\mathcal{H}}\left[1-\frac{e^{2}}{24 \pi^{2}} \log \frac{e^{2} \overrightarrow{\mathcal{H}}^{2}}{\mu^{4}}\right]=\mu_{\text {vac }} \overrightarrow{\mathcal{H}}$

and the QED vacuum responds to the background magnetic field as a diamagnet with the magnetic permeability of the following form:

$\mu_{v a c}=1-\frac{e^{2}}{24 \pi^{2}} \log \left(\frac{e^{2} \overrightarrow{\mathcal{H}}^{2}}{\mu^{4}}\right)<1$ diamagnetic.

The diamagnetism of the QED vacuum means that it repels the magnetic fields by forming induced magnetic field in the direction opposite to that of the applied magnetic field. This phenomenon is similar to the Landau diamagnetism of free electron gas when the counteracting field is formed when the electron trajectories are curved due to the Lorentz force. This also can be seen from the vacuum energy expression (see Fig. 1):

$\epsilon=\frac{\overrightarrow{\mathcal{H}}^{2}}{2}-\frac{e^{2} \overrightarrow{\mathcal{H}}^{2}}{48 \pi^{2}}\left[\log \left(\frac{e^{2} \overrightarrow{\mathcal{H}}^{2}}{\mu^{4}}\right)-1\right]$.

In the case of pure electric field the one-loop Lagrangian has the following form:

$$
\begin{aligned}
\mathcal{L}^{(1)}= & -\frac{\mu^{4}}{8 \pi^{2}} \int_{0}^{\infty} \frac{d s}{s^{3}} \\
& \times\left(\frac{b s \cos (b s)}{\sin (b s)}-1-\frac{b^{2} s}{2}\left(\frac{\cos s}{\sin s}-\frac{s}{\sin ^{2} s}\right)\right),
\end{aligned}
$$

where

$$
b^{2}=-2 e^{2} \mathcal{F} / \mu^{4}=e^{2} \overrightarrow{\mathcal{E}}^{2} / \mu^{4}, \quad \mathcal{G}=0,
$$

and has singularities at $s=s_{n}=\pi n / b=\mu^{2} \pi n / e \mathcal{E}$. The integration path is considered to lie above the real axis, therefore we shall obtain a large positive imaginary contribution to $\mathcal{L}^{(1) 4}$ :

$2 \operatorname{Im} \mathcal{L}^{(1)}=\frac{e^{2} \mathcal{E}^{2}}{4 \pi^{3}} \sum_{n=1}^{\infty} \frac{1}{n^{2}}=\frac{e^{2} \mathcal{E}^{2}}{24 \pi}$.

The real part of the Lagrangian in the electric field is

$\operatorname{Re}^{(1)}=-\frac{e^{2} \overrightarrow{\mathcal{E}}^{2}}{48 \pi^{2}}\left[\log \left(\frac{e^{2} \overrightarrow{\mathcal{E}}^{2}}{\mu^{4}}\right)-1\right]$.

The formulas (2.16), (2.23) and (2.22) prove that the effective Lagrangian is the analytical function of the variable $\mathcal{F}$ and has the general form (2.16). The corresponding energy density takes the following form:

$\epsilon=\frac{\overrightarrow{\mathcal{E}}^{2}}{2}-\frac{e^{2} \overrightarrow{\mathcal{E}}^{2}}{48 \pi^{2}}\left[\log \left(\frac{e^{2} \overrightarrow{\mathcal{E}}^{2}}{\mu^{4}}\right)+1\right]$

and its behaviour is similar to the one shown on Fig. 1. The electric permeability $\overrightarrow{\mathcal{D}}=\frac{\partial \mathcal{L}}{\partial \overrightarrow{\mathcal{E}}}=\varepsilon \overrightarrow{\mathcal{E}}$, where

$\varepsilon=1-\frac{e^{2}}{24 \pi^{2}} \log \left(\frac{e^{2} \overrightarrow{\mathcal{E}}^{2}}{\mu^{4}}\right)+i \frac{e^{2}}{24 \pi}$.

In the next section we shall consider the renormalisation group invariant derivation of the all-loop effective Lagrangian (2.1) and the generalised expressions for the magnetic induction (2.17) and permeability (2.18) as well as the electromagnetic energy-momentum tensor and its trace.

\section{Renormalisation group equation for effective Lagrangian}

Let us derive the exact expression of the effective Lagrangian by using the renormalisation group equation $[15,16]$. The effective action $\Gamma$ is renormalisation group invariant quantity:

$$
\begin{aligned}
\Gamma= & \sum_{n} \int d x_{1} \ldots d x_{n} \Gamma_{\mu_{1} \ldots \mu_{n}}^{(n) a_{1} \ldots a_{n}}\left(x_{1}, \ldots, x_{n}\right) A_{\mu_{1}}^{a_{1}}\left(x_{1}\right) \\
& \ldots A_{\mu_{n}}^{a_{n}}\left(x_{n}\right),
\end{aligned}
$$

because the vertex functions and gauge fields transforms as follows:

$$
\begin{aligned}
\Gamma_{r}^{(n) a_{1} \ldots a_{n}} & =Z_{3}^{n / 2} \Gamma_{u n}^{(n)} a_{\mu_{n}}^{a} a_{1} \ldots a_{n} \\
\mu_{1} \ldots \mu_{n} & \\
A_{\mu}^{a}(x)_{r} & =Z_{3}^{-1} A_{\mu}^{a}(x)_{u n}, \quad g_{r}=Z_{3}^{1 / 2} g_{u n} .
\end{aligned}
$$

\footnotetext{
4 The universal character of the electric instability of the vacuum was discussed in the recent article [84].
} 
The renormalisation group equation takes the form

$$
\left\{\mu^{2} \frac{\partial}{\partial \mu^{2}}+\beta(g) \frac{\partial}{\partial g}+\gamma(g) \int d^{4} x A_{\mu}^{a}(x) \frac{\delta}{\delta A_{\mu}^{a}(x)}\right\} \Gamma=0,
$$

where $\beta(g)$ is the Callan-Symanzik beta function, the $\gamma(g)$ is the anomalous dimension. When $\mathcal{G}=\overrightarrow{\mathcal{E}} \overrightarrow{\mathcal{H}}=0$ it reduces to the form

$\left\{\mu^{2} \frac{\partial}{\partial \mu^{2}}+\beta(g) \frac{\partial}{\partial g}+2 \gamma(g) \mathcal{F} \frac{\partial}{\partial \mathcal{F}}\right\} \mathcal{L}=0$,

where in the covariant background gauge $\beta=-g \gamma$ [12]. By introducing a dimensionless quantity

$\mathcal{M}(g, t)=\frac{\partial \mathcal{L}}{\partial \mathcal{F}}, \quad t=\frac{1}{2} \ln \left(2 g^{2} \mathcal{F} / \mu^{4}\right)$

one can get

$\left\{-\frac{\partial}{\partial t}+\bar{\beta}(g) \frac{\partial}{\partial g}+2 \bar{\gamma}(g)\right\} \mathcal{M}(g, t)=0$,

where

$\bar{\gamma}=\frac{\gamma}{1-\gamma}, \quad \bar{\beta}=\frac{\beta}{1-\gamma}$

and (2.12) plays the role of the boundary condition:

$\mathcal{M}(g, 0)=-1$.

From Eqs. (3.2) and (3.4) it follows that

$\bar{\gamma}=-\left.\frac{1}{2} \frac{\partial \mathcal{M}(g, t)}{\partial t}\right|_{t=0}, \quad \bar{\beta}=\left.\frac{1}{2} g \frac{\partial \mathcal{M}(g, t)}{\partial t}\right|_{t=0}$.

The solution of the renormalisation group equation (3.2) in terms of effective coupling constant $\bar{g}(g, t)$, with the boundary condition $\bar{g}(g, 0)=g$, has the following form $[15,16]$ :

$\frac{\partial \mathcal{L}}{\partial \mathcal{F}}=-\frac{g^{2}}{\bar{g}^{2}(t)}, \quad \frac{d \bar{g}}{d t}=\bar{\beta}(\bar{g})$.

The behaviour of the effective Lagrangian at large fields is similar to the behaviour of the gauge theory at large momentum. It follows that $\mathcal{M}(g, t)$ is completely determined for all $t$ in terms of its first derivative (3.5) at $t=0$. To define the effective Lagrangian $\mathcal{L}$ one should perform additional integration, which we shall do in the next section.

The above results allow to obtain renormalisation group expressions for the physical quantities considered above in one-loop approximation. Indeed, with these expressions in hand we can calculate different observables of physical interest, that will include the effective energy momentum tensor, vacuum energy density, the magnetic permeability, the effective coupling constants and their behaviour as functions of the external fields.

\section{Massless QED}

By using the one loop expression (2.16) derived above one can calculate the derivative

$\mathcal{M}(t, e)=\frac{\partial \mathcal{L}}{\partial \mathcal{F}}=-1+\frac{e^{2}}{24 \pi^{2}} \ln \frac{2 e^{2} \mathcal{F}}{\mu^{4}}, \quad \mathcal{G}=0$,

and the Callan-Symanzik beta function (3.5) takes the following form:

$\bar{\beta}=\left.\frac{1}{2} e \frac{\partial \mathcal{M}}{\partial t}\right|_{t=0}=\frac{1}{24 \pi^{2}} e^{3}$.

The effective coupling constant (3.6) in the one-loop approximation is

$\bar{e}^{2}\left(\overrightarrow{\mathcal{H}}^{2}\right)=\frac{e^{2}}{1-\frac{e^{2}}{24 \pi^{2}} \log \left(\frac{e^{2} \overrightarrow{\mathcal{H}}^{2}}{\mu^{4}}\right)}$

and tends to infinity at the magnetic field

$e^{2} \mathcal{H}_{0}^{2}=\mu^{4} e^{\frac{24 \pi^{2}}{e^{2}}}$

In order to estimate the value of the critical field one can consider the mass parameter $\mu$ to be of the order of the electron mass $m$. Then one can get

$$
\begin{aligned}
H_{0}^{2} & =\left(\frac{m^{2} c^{3}}{e \hbar}\right)^{2} \exp \left(\frac{24 \pi^{2}}{e^{2} / \hbar c}\right)=H_{c}^{2} \exp \left(\frac{24 \pi^{2}}{e^{2} / \hbar c}\right), \\
\alpha & =\frac{e^{2}}{4 \pi \hbar c},
\end{aligned}
$$

where the critical field $H_{c}$ is

$H_{c}=\frac{m^{2} c^{3}}{e \hbar} \approx 4.410^{13}$ Gauss .

The perturbation expansion breaks down at the "Moscow zero" shown on Fig. 1.

As far as the derivative (3.6) of the effective Lagrangian (3.1) has transparent expression in terms of the effective coupling constant (3.6) one can obtain the effective Lagrangian by integration over $\mathcal{F}$ :

$\mathcal{L}(\mathcal{F})=\int \frac{\partial \mathcal{L}}{\partial \mathcal{F}} d \mathcal{F}=-\int \frac{g^{2}}{\bar{g}^{2}(t)} d \mathcal{F}$.

By using the relation (3.1) to express the differential $g^{2} d \mathcal{F}=$ $\mu^{4} e^{2 \tau} d \tau$ through $d \tau$ one can represent the Lagrangian in the form:

$\mathcal{L}(\mathcal{F})=-\mu^{4} \int \frac{e^{2 \tau}}{\bar{g}^{2}(\tau)} d \tau, \quad t=\frac{1}{2} \ln \left(2 g^{2} \mathcal{F} / \mu^{4}\right)$.

In massless QED the magnetic induction (2.17) will take the following form:

$\overrightarrow{\mathcal{B}}=-\frac{\partial \mathcal{L}}{\partial \overrightarrow{\mathcal{H}}}=-\frac{\partial \mathcal{L}}{\partial \mathcal{F}} \overrightarrow{\mathcal{H}}=\mu_{\text {vac }} \overrightarrow{\mathcal{H}}$ 
Therefore the vacuum permeability (2.18) can be expressed through the effective coupling constant $\bar{e}^{2}(t)$ :

$\mu_{v a c}=\frac{e^{2}}{\bar{e}^{2}(t)}$.

The effective Lagrangian approach allows to calculate the quantum-mechanical corrections to the energy momentum tensor by using the formula derived by Schwinger in [5]:

$$
\begin{aligned}
T_{\mu \nu}= & -g_{\mu \nu} \mathcal{L}+\frac{\partial \mathcal{L}}{F_{\mu \lambda}} F_{\nu \lambda}=-g_{\mu \nu} \mathcal{L}+F_{\mu \lambda} F_{\nu \lambda} \frac{\partial \mathcal{L}}{\partial \mathcal{F}} \\
& +g_{\mu \nu} \frac{\partial \mathcal{L}}{\partial \mathcal{G}} \mathcal{G} \\
= & \left(F_{\mu \lambda} F_{\nu \lambda}-g_{\mu \nu} \frac{1}{4} F_{\lambda \rho}^{2}\right) \frac{\partial \mathcal{L}}{\partial \mathcal{F}} \\
& -g_{\mu \nu}\left(\mathcal{L}-\mathcal{F} \frac{\partial \mathcal{L}}{\partial \mathcal{F}}-\mathcal{G} \frac{\partial \mathcal{L}}{\partial \mathcal{G}}\right) .
\end{aligned}
$$

In our case, when $\mathcal{G}=0$, we shall find all-loop expression for $T_{\mu \nu}$ by using (4.6):

$$
\begin{aligned}
T_{\mu \nu}= & -\left(F_{\mu \lambda} F_{\nu \lambda}-g_{\mu \nu} \frac{1}{4} F_{\lambda \rho}^{2}\right) \frac{e^{2}}{\bar{e}^{2}(t)} \\
& +g_{\mu \nu}\left(\int \frac{e^{2 t}}{\bar{e}^{2}(t)} d t-\frac{1}{2} \frac{e^{2 t}}{\bar{e}^{2}(t)}\right) \mu^{4} .
\end{aligned}
$$

And for the vacuum energy density we shall get:

$\varepsilon=T_{00}=\frac{\overrightarrow{\mathcal{H}}^{2}}{2} \frac{e^{2}}{\bar{e}^{2}(t)}+\frac{1}{4} T_{\mu \mu}=\mu^{4} \int \frac{e^{2 t}}{\bar{e}^{2}(t)} d t$,

where the trace of the energy momentum tensor $T_{\mu \mu}$ is not equal to zero and characterises the breaking of conformal symmetry in massless QED:

$T \equiv T_{\mu \mu}=4\left(\mathcal{F} \frac{\partial \mathcal{L}}{\partial \mathcal{F}}-\mathcal{L}\right)=4 \mu^{4}\left(\int \frac{e^{2 t}}{\bar{e}^{2}(t)} d t-\frac{1}{2} \frac{e^{2 t}}{\bar{e}^{2}(t)}\right)$,

where $\mathcal{G}=0, \quad t=\frac{1}{2} \ln \left(2 e^{2} \mathcal{F} / \mu^{4}\right)$. It is also useful to obtain the derivative of $T$ expressed in terms of the effective coupling constant:

$\frac{\partial T}{\partial \mathcal{F}}=4 \mathcal{F} \frac{\partial \mathcal{L}}{\partial \mathcal{F}^{2}}=4 \mathcal{F} \frac{\partial \mathcal{M}}{\partial \mathcal{F}}=2 \frac{\partial \mathcal{M}}{\partial t}=-2 \frac{\partial}{\partial t} \frac{e^{2}}{\bar{e}^{2}(t)}$,

and by using (3.6) we shall get

$\frac{\partial T}{\partial \mathcal{F}}=4 e^{2} \frac{\bar{\beta}(\bar{e})}{\bar{e}^{3}}$.

The integration of (4.13) over $\mathcal{F}$ provides the alternative forms of (4.12):

$$
\begin{aligned}
T & =4 \mu^{4} \int_{-\infty}^{t} \frac{e^{2 t} \bar{\beta}(\bar{e}(t))}{\bar{e}^{3}(t)} d t=4 \mu^{4} \int \frac{e^{2 t} d \bar{e}(t)}{\bar{e}^{3}(t)} \\
& =-2 \mu^{4} \int e^{2 t} d \frac{1}{\bar{e}^{2}(t)} .
\end{aligned}
$$

The last two formulas (4.12) and (4.13) provide the all-loop expressions for the conformal anomaly in QED in the massless limit. If one considers the approximation in which $\bar{e}(t)$ is field independent $\bar{e}(t) \equiv e$ then (4.12), (4.13) will reduce to the expression $T=2 \frac{\beta(g)}{g} \mathcal{F}$ given in literature [56-59].

For the one-loop energy momentum tensor (4.9) we shall get

$T_{\mu \nu}=T_{\mu \nu}^{e l}\left[1-\frac{e^{2}}{24 \pi^{2}} \ln \frac{2 e^{2} \mathcal{F}}{\mu^{4}}\right]+g_{\mu \nu} \frac{e^{2}}{24 \pi^{2}} \mathcal{F}, \quad \mathcal{G}=0$,

where we used the expressions (4.1). The energy density and the trace of the energy momentum tensor are

$T_{00}=\frac{\overrightarrow{\mathcal{H}}^{2}}{2}\left(1-\frac{e^{2} \overrightarrow{\mathcal{H}}^{2}}{24 \pi^{2}} \log \frac{e^{2} \overrightarrow{\mathcal{H}}^{2}}{\mu^{4}}\right)+\frac{e^{2}}{24 \pi^{2}} \frac{\overrightarrow{\mathcal{H}}^{2}}{2}$,

$T_{\mu \mu}=\frac{e^{2}}{6 \pi^{2}} \frac{\overrightarrow{\mathcal{H}}^{2}}{2}$

and they represent the one-loop approximation of (4.11) and (4.12). In the next section we shall consider the behaviour of the effective Lagrangian in Yang-Mills theory and QCD.

\section{Effective Lagrangian of Yang-Mills theory}

The loop expansion of the effective action in Yang-Mills theory has the following form:

$$
\begin{aligned}
\Gamma= & \sum_{n} \int d x_{1} \ldots d x_{n} \Gamma_{\mu_{1} \ldots \mu_{n}}^{(n) a_{1} \ldots a_{n}}\left(x_{1}, \ldots, x_{n}\right) A_{\mu_{1}}^{a_{1}}\left(x_{1}\right) \\
& \ldots A_{\mu_{n}}^{a_{n}}\left(x_{n}\right) \\
= & S_{Y M}+W^{(1)}+W^{(2)}+\cdots,
\end{aligned}
$$

and the one-loop effective Lagrangian has the form [7-14]

$$
\begin{aligned}
W^{(1)}= & S_{Y M}(A)+\frac{i}{2} \operatorname{Tr} \ln \left[\frac{\delta^{2} S_{Y M}(A)}{\delta A \delta A}\right] \\
& -i \operatorname{Tr} \ln \left[\nabla_{\mu}(A) \nabla_{\mu}(A)\right],
\end{aligned}
$$

where

$$
\begin{aligned}
S_{Y M}(A)= & -\frac{1}{4} \int d^{4} x \operatorname{tr} G_{\mu \nu} G_{\mu \nu}, \\
G_{\mu \nu}= & \partial_{\mu} A_{\nu}-\partial_{\nu} A_{\mu}-i g\left[A_{\mu}, A_{\mu}\right] \\
H_{\mu \nu}(\alpha)= & \frac{\delta^{2} S_{Y M}(A)}{\delta A \delta A}=\eta_{\mu \nu} \nabla_{\sigma}(A) \nabla_{\sigma}(A)-2 g G_{\mu \nu} \\
& +(\alpha-1) \nabla_{\mu}(A) \nabla_{\nu}(A), \\
H_{F P}= & \nabla_{\mu}(A) \nabla_{\mu}(A) .
\end{aligned}
$$

By using proper time representation we shall get the effective action in the following form: 


$$
\begin{aligned}
\Gamma(A)= & S_{Y M}(A)-\frac{i}{2} \int_{0}^{\infty} \frac{d s}{s} \operatorname{Tr} e^{-i H(\alpha) s} \\
& +i \int_{0}^{\infty} \frac{d s}{s} \operatorname{Tr} e^{-i H_{F P} s}
\end{aligned}
$$

and for the effective Lagrangian the following expression:

$$
\begin{aligned}
\mathcal{L}_{e f f}= & \mathcal{L}_{Y M}-\frac{i}{2} \int_{0}^{\infty} \frac{d s}{s} \operatorname{Tr}(x|U(s)| x) \\
& +i \int_{0}^{\infty} \frac{d s}{s} \operatorname{Tr}\left(x\left|U_{0}(s)\right| x\right)
\end{aligned}
$$

where $U(s)=e^{-i H(\alpha) s}, U_{0}(s)=e^{-i H_{F P} s}$. The Green function in the background field has the following form:

$G(x, y ; A)=-i \int_{0}^{\infty} d s(x|U(s)| y)$.

As is was proven in $[12,13]$, the $\mathcal{L}_{\text {eff }}$ is $\alpha$ independent functional on the solutions of the YM classical equations. On the covariantly constant gauge field solution [10-13]

$A_{\mu}^{a}=-\frac{1}{2} G_{\mu \nu} x_{\nu} n^{a}, \quad n^{2}=1, \quad x_{\mu} A_{\mu}^{a}=0$

the matrix elements can be calculated and have the following form $[12,13]$ :

$$
\begin{aligned}
(x|U(s)| y)= & \frac{i}{(4 \pi s)^{2}} \exp \left\{-\frac{i}{4}(x-y) K(s)(x-y)\right. \\
& \left.+\frac{i}{2} x N y-L(s)+2 N s\right\} \\
\left(x\left|U_{0}(s)\right| y\right)= & \frac{i}{(4 \pi s)^{2}} \exp \left\{-\frac{i}{4}(x-y) K(s)(x-y)\right. \\
& \left.+\frac{i}{2} x N y-L(s)\right\},
\end{aligned}
$$

where the corresponding matrices are

$$
\begin{aligned}
N & =i g G \\
K(s) & =N \operatorname{coth}(N s) \\
L(s) & =\frac{1}{2} \operatorname{tr} \ln [(N s) \sinh (N s)]
\end{aligned}
$$

and

$$
\begin{aligned}
\mathcal{L}^{(1)}= & -\frac{1}{32 \pi^{2}} \int \frac{d s}{s^{3}} \operatorname{Tr} \exp \{-L(s)+2 N s\} \\
& +\frac{1}{16 \pi^{2}} \int \frac{d s}{s^{3}} \operatorname{Tr} \exp \{-L(s)\} .
\end{aligned}
$$

By substituting the matrix elements and calculating the traces one can get $[12,13]$ :

$$
\begin{aligned}
\mathcal{L}^{(1)}= & -\frac{1}{8 \pi^{2}} \int \frac{d s}{s^{3}} e^{-i \mu^{2} s} \frac{\left(g F_{1} s\right)\left(g F_{2} s\right)}{\sinh \left(g F_{1} s\right) \sinh \left(g F_{2} s\right)}- \\
- & \frac{1}{4 \pi^{2}} \int \frac{d s}{s^{3}} e^{-i \mu^{2} s}\left(g F_{1} s\right)\left(g F_{2} s\right) \\
& \times\left[\frac{\sinh \left(g F_{1} s\right)}{\sinh \left(g F_{2} s\right)}+\frac{\sinh \left(g F_{2} s\right)}{\sinh \left(g F_{1} s\right)}\right],
\end{aligned}
$$

where

$$
F_{1}^{2}=-\mathcal{F}-\left(\mathcal{F}^{2}+\mathcal{G}^{2}\right)^{1 / 2}, \quad F_{2}^{2}=-\mathcal{F}+\left(\mathcal{F}^{2}+\mathcal{G}^{2}\right)^{1 / 2}
$$

The first integral here coincides, up to the coefficient 2, with the expression of the one-loop Lagrangian in the scalar electrodynamics. The doubling of this expression is associated with the additional degrees of freedom due to the vector bosons isospin. The second term is due to the spin contribution $-2 g G_{\mu \nu}$ in the operator $H_{\mu \nu}$. We introduced the mass parameter $\mu^{2}$ in order to control the infrared singularities and to make the integrals convergent at infinity [12]. Still, this is not enough to make integrals convergent at infinity. By using the real eigenvalues

$f_{1}^{2}=\mathcal{F}+\left(\mathcal{F}^{2}+\mathcal{G}^{2}\right)^{1 / 2}, \quad f_{2}^{2}=-\mathcal{F}+\left(\mathcal{F}^{2}+\mathcal{G}^{2}\right)^{1 / 2}$

one can observe that the second term in the square bracket will take the form $\frac{\sinh \left(g f_{2} s\right)}{\sin \left(g f_{1} s\right)}$ and the integral diverges exponentially in the infrared region at infinity. We shall choose the integration counter in the complex plane (s) so as to guarantee the convergence of the last integral. For that one should rotate the integration counter in the third integral by the substitution $s \rightarrow-i s$. The same rotation of the counter can be performed in the first integral as far it is convergent in any way. Thus we shall get $[12,13]$

$$
\begin{aligned}
\mathcal{L}^{(1)}= & \frac{1}{8 \pi^{2}} \int_{0}^{\infty} \frac{d s}{s^{3}} e^{-\mu^{2} s} \frac{\left(g f_{1} s\right)\left(g f_{2} s\right)}{\sinh \left(g f_{1} s\right) \sin \left(g f_{2} s\right)} \\
& +\frac{1}{4 \pi^{2}} \int_{0}^{\infty} \frac{d s}{s} e^{-i \mu^{2} s}\left(g f_{1}\right)\left(g f_{2}\right) \frac{\sin \left(g f_{1} s\right)}{\sinh \left(g f_{2} s\right)} \\
& -\frac{1}{4 \pi^{2}} \int_{0}^{\infty} \frac{d s}{s} e^{-\mu^{2} s}\left(g f_{1}\right)\left(g f_{2}\right) \frac{\sin \left(g f_{2} s\right)}{\sinh \left(g f_{1} s\right)}
\end{aligned}
$$

The integrals are still diverging in the ultraviolet region at the $s=0$. In order to renormalise the Lagrangian we have to identify the ultraviolet divergences in the above integrals. These are

$$
\begin{aligned}
& \frac{\left(g f_{1} s\right)\left(g f_{2} s\right)}{\sinh \left(g f_{1} s\right) \sin \left(g f_{2} s\right)}=1-\frac{g^{2}}{6}\left(f_{1}^{2}-f_{2}^{2}\right) s^{2}+\mathcal{O}\left(s^{4}\right) \\
& f_{1} f_{2} \frac{\sin \left(g f_{1} s\right)}{\sinh \left(g f_{2} s\right)}=f_{1}^{2}+\mathcal{O}\left(s^{2}\right) \\
& f_{1} f_{2} \frac{\sin \left(g f_{2} s\right)}{\sinh \left(g f_{1} s\right)}=f_{2}^{2}+\mathcal{O}\left(s^{2}\right) .
\end{aligned}
$$

Subtracting these terms, which are quadratic in the field strength tensor, we shall get the renormalised effective Lagrangian [12]: 


$$
\begin{aligned}
\mathcal{L}^{(1)}= & \frac{1}{8 \pi^{2}} \int_{0}^{\infty} \frac{d s}{s^{3}} e^{-\mu^{2} s} \\
& \times\left(\frac{\left(g f_{1} s\right)\left(g f_{2} s\right)}{\sinh \left(g f_{1} s\right) \sin \left(g f_{2} s\right)}-1+\frac{1}{6}(g s)^{2}\left(f_{1}^{2}-f_{2}^{2}\right)\right) \\
& +\frac{g^{2}}{4 \pi^{2}} \int_{0}^{\infty} \frac{d s}{s} e^{-i \mu^{2} s}\left(f_{1} f 2 \frac{\sin \left(g f_{1} s\right)}{\sinh \left(g f_{2} s\right)}-f_{1}^{2}\right) \\
& -\frac{g^{2}}{4 \pi^{2}} \int_{0}^{\infty} \frac{d s}{s} e^{-\mu^{2} s}\left(f_{1} f_{2} \frac{\sin \left(g f_{2} s\right)}{\sinh \left(g f_{1} s\right)}-f_{2}^{2}\right)
\end{aligned}
$$

Now the integrals are convergent in both regions, in the infrared and in the ultraviolet. First let us consider a pure chromomagnetic field:

$\mathcal{G}=0, \quad \mathcal{F}=\frac{\overrightarrow{\mathcal{H}}_{a}{ }^{2}-\overrightarrow{\mathcal{E}}_{a}{ }^{2}}{2}>0, \quad f_{1}^{2}=2 \mathcal{F}, \quad f_{2}^{2}=0$.

The Lagrangian (5.16) will take the form

$$
\begin{aligned}
\mathcal{L}^{(1)}= & +\frac{1}{8 \pi^{2}} \int_{0}^{\infty} \frac{d s}{s^{3}} e^{-\mu^{2} s}\left(\frac{g f_{1} s}{\sinh \left(g f_{1} s\right)}-1+\frac{1}{6}(g s)^{2} f_{1}^{2}\right) \\
& +\frac{g^{2}}{4 \pi^{2}} \int_{0}^{\infty} \frac{d s}{s} e^{-i \mu^{2} s}\left(\frac{f_{1} \sin \left(g f_{1} s\right)}{g s}-f_{1}^{2}\right) .
\end{aligned}
$$

At strong chromomagnetic fields $g^{2} \overrightarrow{\mathcal{H}}_{a}^{2} \geq \mu^{4}$ the asymptotic behaviour of the real part is [12]

$$
\begin{aligned}
\Re \mathcal{L}^{(1)} & \approx+\frac{g^{2}}{48 \pi^{2}} \frac{\overrightarrow{\mathcal{H}}_{a}^{2}}{2} \ln \frac{g^{2} \overrightarrow{\mathcal{H}}_{a}^{2}}{\mu^{4}}-\frac{g^{2}}{4 \pi^{2}} \frac{\overrightarrow{\mathcal{H}}_{a}^{2}}{2} \ln \frac{g^{2} \overrightarrow{\mathcal{H}}_{a}^{2}}{\mu^{4}} \\
& =-\frac{11 g^{2}}{48 \pi^{2}} \frac{\overrightarrow{\mathcal{H}}_{a}^{2}}{2} \ln \frac{g^{2} \overrightarrow{\mathcal{H}}_{a}^{2}}{\mu^{4}},
\end{aligned}
$$

where the first term represents the diamagnetism, which counteracts to the external field caused by the quantum current induced by the charged vector bosons in the vacuum and the second term represents the paramagnetism, an effect associated with the polarisation of the gluon spins, which, as one can see, dominates the asymptotic behaviour [12]. The imaginary part of the effective Lagrangian (5.17) in background chromomagnetic field in our regularisation scheme vanishes [12]:

$$
\begin{aligned}
\Im \mathcal{L}^{(1)}= & -\frac{g f_{1}}{4 \pi^{2}} \int_{0}^{\infty} \frac{d s}{s^{2}} \sin \left(\mu^{2} s\right) \sin \left(g f_{1} s\right) \\
& +\frac{g^{2} f_{1}^{2}}{4 \pi^{2}} \int_{0}^{\infty} \frac{d s}{s} \sin \left(\mu^{2} s\right) \\
= & -\frac{g f_{1}}{4 \pi^{2}} \frac{\pi}{2} g f_{1}+\frac{g^{2} f_{1}^{2}}{4 \pi^{2}} \frac{\pi}{2}=-\frac{g^{2} f_{1}^{2}}{8 \pi}+\frac{g^{2} f_{1}^{2}}{8 \pi}=0,
\end{aligned}
$$

where $f_{1}=\sqrt{\overrightarrow{\mathcal{H}}_{a}^{2}}=\mathcal{H}$. A similar conclusion was derived in [50] by using alternative regularisation. The significance of the absence/presence of the imaginary part in the effective Lagrangian connected with the fact that it defines the quantum-mechanical stability of a given field configuration. The above conclusion on the absence of the imaginary part is not conclusive due to the tachyonic eigenmode in the operator $(5.3)[35,36,97]$, and we shall discuss the stability of the background field configurations in the subsequent Sect. 8 . Here we shall refer to the articles of Leutwyler [46,47] and Flory $[48,49]$, where they come to the same conclusion that there is no imaginary part in the effective Lagrangian in chromomagnetic field. Leutwyler was considering the selfdual chromomagnetic background field configurations and demonstrated that there is no imaginary part in the effective Lagrangian $[46,47]$ and that the real part of the effective Lagrangian has the form identical to (6.4). Flory included the quartic self-interaction of eigenmodes and also came to the same conclusion [52]. The physical reason behind this universality lies in the fact that even when the background field depends on space-time coordinates the part of the effective Lagrangian $\overline{\mathcal{L}}(\mathcal{F}, \mathcal{G})$ which depends only on the field strength tensor but not of its covariant derivatives has a universal form $[12,15,16]$. In other words, as far as the wavelength of the fluctuating fields is very long, the effective action is not sensitive to the fine structure of the fluctuating fields.

Let us now consider pure chromoelectric fields $\mathcal{G}=$ $0, \mathcal{F}<0$ and $f_{1}^{2}=0, f_{2}^{2}=-2 \mathcal{F}$ :

$$
\begin{aligned}
\mathcal{L}^{(1)}= & \frac{1}{8 \pi^{2}} \int_{0}^{\infty} \frac{d s}{s^{3}}\left(\frac{\left(g f_{2} s\right)}{\sin \left(g f_{2} s\right)}-1+\frac{1}{3}(g s)^{2} \mathcal{F}\right) \\
& -\frac{g^{2}}{4 \pi^{2}} \int_{0}^{\infty} \frac{d s}{s}\left(f_{2} \frac{\sin \left(g f_{2} s\right)}{g s}-f_{2}^{2}\right) .
\end{aligned}
$$

The Lagrangian has singularities on the real axis at $s_{n}=$ $n \pi / e \mathcal{E}$, and the integration path is considered to lie above the real axis $[12,13]$ :

$2 \operatorname{Im} \mathcal{L}^{(1)}=\frac{(g \mathcal{E})^{2}}{4 \pi^{3}} \sum_{n=1}^{\infty} \frac{(-1)^{n+1}}{n^{2}}=\frac{g^{2} \mathcal{E}^{2}}{48 \pi}$

This is the probability per unit time and per unit volume that gluons are created by the chromoelectric field. Due to the masslessness of QCD gluons the above formula does not contain the Sauter-Schwinger exponentially small tunnelling factor $\exp \left(-\pi \frac{m^{2} c^{3}}{e \hbar \mathcal{E}}\right)$ and even a weak chromoelectric field will break down creating a cloud of soft gluons from the vacuum neutralising the imposed colour electric field. While the exact results for the imaginary part of the effective action (5.20) depend on the details of the background field, it was argued in $[84,85]$ that the threshold singularity is universal. The physical reason for this universality lies in the fact that the onset of pair production is dominated by the long-range fluctuations of the particles created from the vacuum and becomes insensitive to the details of the field profile $[84,85]$.

The infrared long wavelength gluons created from the vacuum are strongly interacting. In the one-loop approximation the interaction between the produced gluons is not 
considered. In $[33,34]$ the authors considered a semiclassical corrections to the production rates due to the interactions between created pairs and suggested a mechanism of colour neutralisation.

By considering a cylindrical non-homogeneous chromoelectric field configurations in [32] Flory demonstrated a possible formation of a chromoelectric flux tube of a finite radius between quark-antiquark pairs which are embedded into the chromomagnetic gluon condensate [15] (see the next section). The alternative mechanisms of formation of chromoelectric and chromomagnetic flux tubes were considered in [31] and in [30].

\section{Chromomagnetic gluon condensate}

Let us now apply the renormalisation condition (2.12) to the Yang-Mills effective Lagrangian (5.16) and (5.17). This leads to the following expression for the renormalised effective Lagrangian in chromomagnetic fields $[12,15]$ :

$$
\begin{aligned}
\mathcal{L}^{(1)}= & \frac{\mu^{4}}{8 \pi^{2}} \int_{0}^{\infty} \frac{d s}{s^{3}}\left(\frac{a s}{\sinh a s}-1-\frac{a^{2} s}{2}\left(\frac{1}{\sinh s}-\frac{s \cosh s}{\sinh ^{2} s}\right)\right) \\
& +\frac{\mu^{4}}{4 \pi^{2}} \int_{0}^{\infty} \frac{d s}{s^{3}}\left(a s \sin (a s)-\frac{a^{2} s}{2}(\sin s+s \cos s)\right),
\end{aligned}
$$

where

$$
\begin{aligned}
& a=g(2 \mathcal{F})^{1 / 2} / \mu^{2}, \quad \mathcal{F}=\frac{1}{4} G_{\mu \nu}^{2}>0, \\
& \mathcal{G}=\frac{1}{4} G_{\mu \nu} G_{\mu \nu}^{*}=0 .
\end{aligned}
$$

The Lorentz and gauge invariant field $\mathcal{F}$ is positive and corresponds to the chromomagnetic field configurations. The proper time integration can be performed exactly by using the integrals presented in Appendix and the one-loop SU(2) Lagrangian in terms of Lorentz and gauge invariant field $\mathcal{F}$ is

$$
\mathcal{L}^{(1)}=-\frac{11}{48 \pi^{2}} g^{2} \mathcal{F}\left(\ln \frac{2 g^{2} \mathcal{F}}{\mu^{4}}-1\right)
$$

and the effective Lagrangian in SU(N) gauge theory will take the following form [15]:

$\mathcal{L}=-\mathcal{F}-\frac{11 N}{96 \pi^{2}} g^{2} \mathcal{F}\left(\ln \frac{2 g^{2} \mathcal{F}}{\mu^{4}}-1\right)$.

As it follows from this expression, the QCD vacuum responds to the background chromomagnetic field by inducing a quantum current of the charged vector bosons which amplifies the chromomagnetic field imposed on the vacuum. The chromomagnetic magnetic induction $\overrightarrow{\mathcal{B}}_{a}$ of the QCD vacuum is
$\overrightarrow{\mathcal{B}}_{a}=-\frac{\partial \mathcal{L}}{\partial \overrightarrow{\mathcal{H}}_{a}}=\overrightarrow{\mathcal{H}}_{a}\left[1+\frac{g^{2} N}{96 \pi^{2}} \log \frac{g^{2} \overrightarrow{\mathcal{H}}_{a}^{2}}{\mu^{4}}\right]=\mu_{\text {vac }} \overrightarrow{\mathcal{H}}_{a}$

The QCD vacuum responds to the background magnetic field as paramagnet with the magnetic permeability of the following form [12]:

$\mu_{\text {vac }}=-\frac{\partial \mathcal{L}}{\partial \mathcal{F}}=1+\frac{g^{2} N}{96 \pi^{2}} \log \frac{g^{2} \overrightarrow{\mathcal{H}}_{a}^{2}}{\mu^{4}}>1$ paramagnetic.

The paramagnetism of the QCD vacuum means that it amplifies the applied chromomagnetic field by generating induced chromomagnetic field in the direction of the applied field. This phenomenon is similar to the Pauli paramagnetism, an effect associated with the polarisation of the electron spins. In QCD the polarisation of the vector boson spins is responsible for this amplification of the background field (5.18). This also can be seen from the vacuum energy density (see Fig. 2).

$\epsilon(\mathcal{F})=\mathcal{F}+\frac{11 N}{96 \pi^{2}} g^{2} \mathcal{F}\left(\ln \frac{2 g^{2} \mathcal{F}}{\mu^{4}}-1\right)$

with its new minimum outside of the perturbative vacuum $<$ $\mathcal{F}>=0$, at the renormalisation group invariant field strength [15]

$\left\langle 2 g^{2} \mathcal{F}\right\rangle_{\text {vac }}=\mu^{4} \exp \left(-\frac{96 \pi^{2}}{11 N g^{2}(\mu)}\right)=\Lambda_{Q C D}^{4}$

characterising the dynamical breaking of conformal symmetry of the SU(N) gauge field theory. ${ }^{5}$ The Lorentz invariant form of the effective action (6.7) suggests that there are many states which have the same energy density as the covariantly constant chromomagnetic field. In a series of articles [64-68] the authors found and explored spatially homogeneous solutions of the YM equations which are invariant with respect to the Lorentz transformations and conveniently represent the gauge field fluctuations in the vacuum. The average $\langle\ldots\rangle$ in (6.8) can be understood as average over these field configurations (see also $[55,62,69,70]$ ).

For the energy momentum tensor (4.9) we shall get

$$
\begin{aligned}
T_{\mu \nu}= & T_{\mu \nu}^{Y M}\left[1+\frac{11 N g^{2}}{96 \pi^{2}} \ln \frac{2 g^{2} \mathcal{F}}{\mu^{4}}\right]-g_{\mu \nu} \frac{11 N}{96 \pi^{2}} g^{2} \mathcal{F}, \\
& \mathcal{G}=0 .
\end{aligned}
$$

The trace of the energy momentum tensor is not equal to zero and characterises the breaking of conformal symmetry in QCD:

\footnotetext{
5 The Lorentz invariant average \langle\rangle over the covariantly constant field $G_{\mu \nu}$ orientations can be performed as in [63], [15]. In [63] the invariant measure was taken in the form $d \mu=d \overrightarrow{\mathcal{H}} d \overrightarrow{\mathcal{E}} \delta\left(\mathcal{F}-\mathcal{F}_{\text {min }}\right) \delta\left(\mathcal{G}^{2}-\mathcal{G}_{\text {min }}^{2}\right)$.
} 
$T=T_{\mu \mu}=-\frac{11 N}{24 \pi^{2}} g^{2} \mathcal{F}$.

The vacuum energy density is given in (6.7): $T_{00}=\epsilon(\mathcal{F})$ with its minimum at (6.8) [15]. Substituting this value into the expression for the energy momentum tensor (6.9) we shall get the expression which is proportional to the metric tensor $g_{\mu \nu}$ :

$\left\langle T_{\mu \nu}\right\rangle_{v a c}=-g_{\mu \nu} \frac{11 N}{96 \pi^{2}}\left\langle g^{2} \mathcal{F}\right\rangle_{v a c}$

and is therefore a relativistically invariant characterisation of the vacuum with its negative energy density $\epsilon_{v a c}=\left\langle T_{00}\right\rangle_{v a c}$ and the pressure $P_{v a c}=-\epsilon_{v a c}$. This is an important result because the vacuum state should be Lorentz invariant and its stress tensor $T_{\mu \nu}$ should be the same in all frames [1720]. As a result, its vacuum average value can only be of the cosmological type $\left\langle T_{\mu \nu}\right\rangle=\epsilon_{v a c} g_{\mu \nu}$, and indeed it is.

Let us consider the behaviour of the effective Lagrangian from the renormalisation group point of view and compare it with the behaviour of the effective coupling constant. The equations derived above are universally true for the nonAbelian field as well. Thus when $\mathcal{G}=\overrightarrow{\mathcal{E}}_{a} \overrightarrow{\mathcal{H}}_{a}=0$ we have

$\mathcal{M}(t, g)=\frac{\partial \mathcal{L}}{\partial \mathcal{F}}=-\frac{g^{2}}{\bar{g}^{2}(t)}, \quad \frac{d \bar{g}}{d t}=\bar{\beta}(\bar{g})$.

The vacuum magnetic permeability introduced in (2.18) will take the following form [12]:

$\mu_{v a c}=\frac{g^{2}}{\bar{g}^{2}(t)}, \quad \mathcal{G}=0$.

The Callan-Symanzik beta function can be calculated by using (6.4):

$\bar{\beta}=\left.\frac{1}{2} g \frac{\partial \mathcal{M}}{\partial t}\right|_{t=0}=-\frac{11 N}{96 \pi^{2}} g^{3}$

and the effective coupling constant as a function of the field has the form

$\bar{g}^{2}(\mathcal{F})=\frac{g^{2}}{1+\frac{11 g^{2} N}{96 \pi^{2}} \ln \frac{2 g^{2} \mathcal{F}}{\mu^{4}}}$,

where we introduced the Casimir operator $C_{2}(G)=N$ for the gauge group $G=S U(N)$.

Let us consider the value of the field strength tensor $\mathcal{F}_{0}$ at which the vacuum energy density (6.7) vanishes $\epsilon\left(\mathcal{F}_{0}\right)=0$, as it is shown on Fig. 2:

$2 g^{2} \mathcal{F}_{0}=\mu^{4} \exp \left(-\frac{96 \pi^{2}}{11 g^{2} N}+1\right)=e\left\langle 2 g^{2} \mathcal{F}\right\rangle_{\text {vac }}$.

The effective coupling constant (6.15) at this field strength has the value

$\bar{g}^{2}\left(\mathcal{F}_{0}\right)=\frac{96 \pi^{2}}{11 N}$.
It follows that the effective coupling constant at the intersection point $\mathcal{F}_{0}$ is small:

$\bar{g}^{2}\left(\mathcal{F}_{0}\right)=\frac{96 \pi^{2}}{11 N} \ll 1$ if $N \gg \frac{96 \pi^{2}}{11}$.

The energy density curve $\epsilon(\mathcal{F})$ (6.7) intersects the horizontal zero energy line at the nonzero angle $\theta$ (see Fig. 2):

$\tan \theta=\frac{11 g^{2} N}{96 \pi^{2}}>0$.

This means that i) the true vacuum state is below the perturbative vacuum and that ii) there is a nonzero chromomagnetic field in the vacuum. Now the question is, how far into the infrared region one can continue the energy density curve by using the perturbative result? Let us consider the fields which are approaching the infrared pole. This can be done, in particular, by using the following parametrisation:

$\mathcal{F}_{n}=e^{1-n}\langle\mathcal{F}\rangle_{\text {vac }}$,

where the parameter $n$ is less than one, and we have $\mathcal{F}_{n} \rightarrow\langle\mathcal{F}\rangle_{\text {vac }}$ when $n$ tends to unity from below. At these fields values the effective coupling constant (6.15) tends to zero:

$\bar{g}^{2}\left(\mathcal{F}_{n}\right)=\frac{96 \pi^{2}}{11 N(1-n)} \rightarrow 0$

if the product $N(1-n) \rightarrow \infty$ is large and the t'Hooft coupling constant $g^{2} N=\lambda$ is fixed and small. It follows then that the effective coupling constant can be made small to justify the use of the perturbative result and the energy density curve can be continuously extended infinitesimally close to the value of the vacuum field $\langle\mathcal{F}\rangle_{\text {vac }}$, as it is shown on Fig. 2. Let us analyse how the field at the intersection point (6.16) and the effective coupling constant (6.17) are changing when we include the two-loop approximation. The two-loop ${ }^{6}$ effective Lagrangian has the form [12]

$$
\begin{aligned}
\mathcal{L}= & -\mathcal{F}-\left(\frac{11}{8(4 \pi)^{2}} g^{2} N+\frac{34}{3(4 \pi)^{4}}\left(g^{2} N\right)^{2}\right) \mathcal{F} \\
& \times\left(\ln \frac{2 g^{2} \mathcal{F}}{\mu^{4}}-1\right) .
\end{aligned}
$$

The field at the intersection point (6.16) is shifted by an exponentially small correction

$2 g^{2} \mathcal{F}_{0}^{\prime}=\exp \left(-\frac{96 \pi^{2}}{11 \lambda} \cdot \frac{1}{1+\frac{17}{33 \pi^{2}} \lambda}+1\right)$.

\footnotetext{
${ }^{6}$ The two-loop beta function (6.12) coefficients $\bar{\beta}=-\beta_{1} g^{3}-\beta_{2} g^{5}+$ $\cdots$ are given by $\beta_{1}=\frac{11 N}{6(4 \pi)^{2}}$ and $\beta_{2}=\frac{34 N^{2}}{6(4 \pi)^{4}}$.
} 
At this field the effective coupling constant is smaller by the factor $1 / 1+\frac{17}{33 \pi^{2}} \lambda$

$$
\bar{g}^{2}\left(\mathcal{F}_{0}^{\prime}\right)=\frac{96 \pi^{2}}{11 N} \cdot \frac{1}{1+\frac{17}{33 \pi^{2}} \lambda} \ll 1,
$$

and the inequality (6.24) is fulfilled at smaller values of $N$ than in the first approximation (6.18). This demonstrates that the one-loop results are stable with respect to the two-loop corrections. The high-loop corrections are analysed below (6.27) by using renormalisation group results (3.6), (6.12).

It is interesting to know if the energy density curve is a continuous function of the field strength $\mathcal{F}$ in the region $\left[0,\langle\mathcal{F}\rangle_{\text {vac }}\right]$ which is outside of the validity of the perturbative calculations and if the energy density curve is a convex function. A non-perturbative functional method developed by Zwanziger in [51] is answering to these questions affirmatively. It seems that further development of his approach can shed even more light to the behaviour of the effective Lagrangian in the non-perturbative region. We already obtained the first derivative of the energy density curve (6.12) and can calculate its second derivative as well:

$$
\frac{\partial \epsilon}{\partial \mathcal{F}}=-\frac{\partial \mathcal{L}}{\partial \mathcal{F}}=\frac{g^{2}}{\bar{g}^{2}}, \quad \mathcal{F} \frac{\partial^{2} \epsilon}{\partial \mathcal{F}^{2}}=\frac{\partial}{\partial t} \frac{g^{2}}{\bar{g}^{2}(t)}=-g^{2} \frac{\bar{\beta}(\bar{g})}{\bar{g}^{3}} .
$$

The sign of the second derivative depends on the sign of the ratio of the beta function to the cube of the effective coupling constant. In QCD, in the perturbative regime this ratio is negative and the second derivative (6.25) is positive:

$\mathcal{F} \frac{\partial^{2} \epsilon}{\partial \mathcal{F}^{2}}=\frac{11}{8(4 \pi)^{2}} g^{2} N+\frac{34}{3(4 \pi)^{4}}\left(g^{2} N\right)^{2}+\cdots$

Thus the energy density curve is convex (see Fig. 2). In QED the overall sign is negative and the energy density curve is concave (see Fig. 1).

Any non-perturbative information about the ratio $\bar{\beta}(\bar{g}) / \bar{g}^{3}$ can be translated into the information about property of the energy density curve. As far as the beta function $\bar{\beta}(g)$ has no zeros, is a negative analytical function of the coupling constant and

$$
\int_{g}^{\infty} \frac{d g}{\bar{\beta}(g)}<\infty
$$

the minimum of the energy density curve is defined by the extremum, where the derivative (6.12) vanishes. By using the expression (6.12) one can derive the value of the chromomagnetic condensate [15]:

$$
\left\langle 2 g^{2} \mathcal{F}\right\rangle_{\text {vac }}=\mu^{4} \exp \left(2 \int_{g}^{\infty} \frac{d g}{\bar{\beta}(g)}\right) .
$$

To all orders in the perturbation theory the derivative of the energy momentum tensor trace can be obtained by using the renormalisation group invariant result (4.13):

$$
\frac{\partial T}{\partial \mathcal{F}}=4 g^{2} \frac{\bar{\beta}(\bar{g}(t))}{\bar{g}(t)^{3}}, \quad t=\frac{1}{2} \ln \left(2 g^{2} \mathcal{F} / \mu^{4}\right) .
$$

Integration over $\mathcal{F}$ gives the trace

$$
T_{\mu \mu}=4 \int \frac{g^{2}}{\bar{g}^{2}} \frac{\bar{\beta}(\bar{g})}{\bar{g}} d \mathcal{F} \text {. }
$$

If one considers the approximation in which the effective coupling constant (3.6) is field independent $\bar{g}(t) \equiv g$ then this formula after integration over $\mathcal{F}$ will reduce to the one given in literature [56-59]:

$T_{\mu \mu}=4 \frac{\bar{\beta}(g)}{g} \mathcal{F}$.

Otherwise the field dependence of the energy momentum trace is defined through the beta functions and effective coupling constant and has more complicated dependence on field strength tensor $\mathcal{F}$.

\section{Effective cosmological constant}

As is follows from (6.11), in the ground state the following relation between energy density $\epsilon_{v a c}$ and pressure $P_{v a c}$ takes place: $\epsilon_{v a c}=-P_{v a c}$. It is a relativistically invariant characterisation of the vacuum [17-20], and it represents a field-theoretical contribution into the effective cosmological constant $\Lambda_{e f f}$ :

$$
\begin{aligned}
\epsilon_{v a c} & =\frac{c^{4}}{8 \pi G} \Lambda_{e f f}=-\frac{b}{192 \pi^{2}}\left\langle 2 g^{2} \mathcal{F}\right\rangle_{v a c} \\
& =-\frac{b}{192 \pi^{2}} \Lambda_{Q C D}^{4} .
\end{aligned}
$$

The chromomagnetic condensate (6.8) is of order $\Lambda_{Q C D}^{4}$, and the vacuum energy density is negative and is about $\epsilon_{v a c} \approx-b 10^{-8} G e V^{4}$. The value of the cosmological constant measured in the observation of the high-z Type Ia supernovae [24-27] and by the Plank Collaboration [28,29] $\epsilon_{\Lambda}=c^{4} \Lambda_{\text {obser }} / 8 \pi G \approx 10^{-47} G e V^{4}$ is about 39 decimal places smaller and positive. It is important to mention that the energy gap depends on a gauge group and a matter content, the beta function (the $b$ parameter in one-loop approximation), as well as of the temperature of the universe [53]. At high temperatures the curve of the effective potential moves upward, the value of the chromomagnetic gluon condensate tends to zero, as well as the $\Lambda_{e f f}$, and the scaling invariance get restored. The phase transition is of the second-order [53].

In the article [74] the authors suggested a possible cancelation mechanism between chromomagnetic and its "mirror chromoelectric" condensates. In this proposal, which 
involves adding to the SM particles a mirror world (dark matter) [76-81], the entire SM is replicated in a mirror world. The new $Z_{2}$ symmetry interchanges SM with the mirror SM, ensuring identical particles and interactions. It is conjectured that the quantum vacua of the "Mirror SM" contribute to the cosmological constant on the same footing as the SM, since mirror particles are expected to gravitate in the same way as the usual ones, and that the mirror chromoelectric gluon condensate contributes to the energy density of the universe with a positive sign and thus may, in principle, eliminates the negative QCD vacuum effect by yielding a cosmological constant small. The alternative mechanism was considered in [75].

In electroweak theory the Higgs vacuum field also generates a negative contribution to the effective cosmological constant [21-23]. In both field theories the value of the $\Lambda_{\text {eff }}$ is of many orders of magnitude larger than the observed value.

\section{Absence of imaginary part in chromomagnet field}

A careful inspection of the charged vector bosons spectrum in a chromomagnetic field by Nielsen, Olesen [35] and Skalozub [36] demonstrated that due to the unstable mode $k_{0}^{2}=k_{\|}^{2}-g f_{1}\left(k_{\|}^{2} \leq g f_{1}\right)$ there is an imaginary part in the effective Lagrangian:

$\operatorname{Im} \mathcal{L}^{(1)}=\operatorname{Im} \frac{g f_{1}}{4 \pi^{2}} \int_{-\infty}^{\infty} d k_{\|} \sqrt{k_{\|}^{2}-g f_{1}-i \epsilon}=-\frac{g^{2} f_{1}^{2}}{8 \pi}$.

In the subsequent publications [37-41,43-45,51-55,103] the theoretical groups at the Niels Bohr Institute, New York University, SLAC and Bari University came to the conclusion that due to the quartic self-interaction term in the YM action there is a hidden Higgs mechanism, which stabilises the system so that the effective Lagrangian remains a real function in the background chromomagnetic field $[38,40,44,51,52]$. This is the reflection of the fact that calculations are performed in the approximation in which only the quadratic term $\delta A_{\mu} H_{\mu \nu} \delta A_{\nu}$ of the fluctuating fields $\delta A_{\mu}$ in the direction of the unstable mode $\Phi$ was taken into the consideration in the loop expansion (5.1) and (5.2). The quartic term $V_{\mu \nu \lambda \rho} \delta A_{\mu} \delta A_{\nu} \delta A_{\lambda} \delta A_{\rho}$ of the Yang-Mills Lagrangian should be taken into consideration in this circumstance and play a crucial role in stabilising the quantum mechanical fluctuations, turning the unstable mode into a stable mode $[31,38,40,44,52]$. The phenomenon is similar to the one in the classical Higgs Lagrangian where the zero field configuration is unstable and a field configuration at the bottom of the potential provides a stable field configuration due to the quartic term. In the pure YM theory the Higgs-like action for the unstable mode was derived in $[31,38,40,44]$. It was proposed to search the stable solutions of the classical YangMills field equations in a fixed background chromo-magnetic field which plays the role of an external order parameter. Without the presence of the order mass parameter, the $g H$ in the given case, the conformal invariance of the pure classical Yang-Mills equations prevents the existence of localised solutions [30]. This program was successfully realised with the discovery of the field configurations which are varying in space due to the development of the unstable mode, the colour magnetic flux tubes and the spaghetti magnetic tubes forming the domain-like field configurations [40]. The configurations are supported by the external chromomagnetic field $g H$. The difficulty here is to calculate the quantum-mechanical fluctuations around these classical field configurations and to see if they remain localised when the external field is switched off. The important conclusion of the investigation was that it pointed out to the fact that the stability of the chromomagnetic field configurations is a natural consequence of the quartic self interaction of the Yang-Mills field. ${ }^{7}$

This result became the initial point for the investigation initiated by Curt Flory in his article devoted to the resolution of the Higgs-like mode problem [52]. His breakthrough idea was to integrate exactly the functional integral over the Higgs-like mode from the start in order to get the quantummechanical contribution to the effective Lagrangian of that mode instead of searching the corresponding classical field configurations. Presenting the amplitude of the Higgs-like mode and of the corresponding action in terms of dimensionless variables $k_{\mu} \rightarrow k_{\mu} / \sqrt{g H}, x_{\mu} \rightarrow x_{\mu} \sqrt{g H}$ one can get $^{8}$ :

$(g H)^{-1 / 2} W=\int \frac{d k_{2}}{2 \pi} e^{-\frac{1}{2}\left(x_{1}+k_{2}\right)^{2}} \Phi_{k_{2}}\left(x_{0}, x_{3}\right)$,

where $\Phi_{k_{2}}\left(x_{0}, x_{3}\right)$ is the dimensionless amplitude of the Higgs-like mode. The action of the Higgs-like mode will take the following form:

$$
\begin{aligned}
& \frac{\text {S}_{\text {higgs mode }}}{\sqrt{2 \pi}} \\
& =\int \frac{d k_{2}}{2 \pi} d x_{0} d x_{3}\left(\left|\partial_{\mu} \Phi_{k_{2}}\right|^{2}+\left|\Phi_{k_{2}}\right|^{2}-\frac{1}{2} g^{2}\right. \\
& \left.\quad \times \int \frac{d p d q}{(2 \pi)^{2}} e^{-\frac{p^{2}+q^{2}}{2}} \Phi_{k_{2}+p}^{*} \Phi_{k_{2}+q}^{*} \Phi_{k_{2}} \Phi_{k_{2}+p+q}\right) .
\end{aligned}
$$

What is essential in this representation is that the dependence on the chromomagnetic field does not show up in the Lagrangian (8.2) and appears only in front of the Higgs-like field amplitude $(g H)^{1 / 2}$ in (8.1). The factor coming from the integration of the action (8.2) over the field $\Phi_{k_{2}}$ is background field independent and does not show up in the renormalised

\footnotetext{
7 A non-perturbative prove of the reality and concavity of the effective action is due to Zwanziger [51].

${ }^{8}$ In the $\mathrm{SU}(2)$ case $W_{\mu}=\frac{1}{\sqrt{2}}\left(A_{\mu}^{1}+A_{\mu}^{2}\right), A_{\mu}=A_{\mu}^{3}$ and $W=W_{1}=$ $-i W_{2}$, as it is defined in $[31,38,40,44]$.
} 
effective Lagrangian. Thus the contribution of the Higgs-like mode to the effective Lagrangian is only through the integration measure and its degeneracy:

$$
\begin{aligned}
& e^{\mathcal{L}_{\text {higgs mode }}} \approx(C g H)^{-\frac{1}{2}\left(\frac{g H}{2 \pi}\right)^{2}} \\
& =\exp \left(-\frac{g^{2} H^{2}}{8 \pi^{2}}(\log g H+\log C)\right),
\end{aligned}
$$

where $C$ is the $g H$ independent value of the functional integral over $\Phi_{k_{2}}\left(x_{0}, x_{3}\right)$. This contribution is a real function of chromomagnetic field [52,53]. After taking into account the contributions from all other modes the effective Lagrangian takes the form which identically coincides with (6.3). This confirms the expression (6.3) being without imaginary part (5.19).

One can consider the above approach of calculating the effective action as an alternative to a standard loop expansion in the following sense: The expansion is organised by rearranging the perturbative expansion (5.1) in a background field $A$ so that the quartic self-interactions of eigenmodes are included into the propagator of the gauge field $G(x, y ; A)$ and the loop expansion is performed in terms of the remaining cubic and cross-mode quartic vertices of the YM action.

\section{Conclusion}

The short overview of the publications devoted to the chromomagnetic gluon condensation and QCD vacuum are given below. The confinement problem from the point of view of the $Q C D$ vacuum and chromomagnetic gluon condensate was considered in the articles of Mandelshatam [92-94], Nambu [95], Adler and Piran [96] and Nielsen and Olesen [42]. The thermodynamics of the Yang-Mills gas by Linde [23]. The publication on generation of galactic magnetic field due to the condensation of vector field was considered in [88]. The induced gravity was considered by Adler [61]. The magnetostatics was considered in [89]. The phenomenology of hadrons and the properties of the QCD vacuum by Shuryak [105]. The mechanism of dynamical supersymmetry breaking and string compactification to four dimension due to the properties of the non-Abelian effective action was suggested by Veneziano and Taylor [104]. The dynamical mass generation in QCD and glueballs spectrum by Cornwall $[98,99]$. The string-like solution of pure YM equations stabilised in the presence of the chromomagnetic condensate by Faddeev and Niemi $[100,101]$ and the monopole condensation by Cho [102]. A complementary to Zwanziger [51] a nonperturbative approach for the construction of effective actions at different scales, the Wilsonian effective actions, was developed by Reuter and Wetterich in series of articles [109-112].

Alternative approach for the investigation of the condensates in Yang-Mills theory is provided by the Monte
Carlo lattice simulations [117-121]. The lattice formulation is offering a non-perturbative regularisation of the YM theory and in principle allows to measure the QCD condensates. One of the aims of these calculations is to extract a nonperturbative value of the vacuum expectation value (VEV) of the composite operator $G_{\mu \nu}^{2}$ :

$\left\langle 0\left|\frac{\alpha_{s}}{\pi} G_{\mu \nu} G_{\mu \nu}\right| 0\right\rangle$.

In perturbation theory this VEV is diverging as the fourth power of the cutoff and after renormalisation is set to zero. In our investigation we were studying a different observable, the effective action $\Gamma[A](1.1)$, which depends on the VEV of the gauge field operator $\left\langle 0\left|A_{\mu}^{a}(x)\right| 0\right\rangle \equiv A_{\mu}^{a}(x)$. As it was stressed in references [117-121], the main difficulty in measuring the condensates of the type (9.1) lies in the necessity to subtract from the Monte Carlo data the dominant perturbative contribution and then to extract the exponentially falling non-perturbative term of the form (6.99), which is the only one of interest from the point of view of the continuum theory. The evaluation of the VEV requires the calculation of the following expression:

$$
\begin{aligned}
& \lim _{a \rightarrow 0} \frac{C}{a^{4}}\langle 0|(1-P)| 0\rangle_{\text {meas }}-\langle 0|(1-P)| 0\rangle_{\text {pert }} \\
& \quad=\left\langle 0\left|\frac{\alpha_{s}}{\pi} G_{\mu \nu} G_{\mu \nu}\right| 0\right\rangle+\cdots,
\end{aligned}
$$

where $P$ is a the plaquette operator and dots denote the operators of higher dimension. The perturbative VEV is represented by a diverging series [113-116]:

$$
\langle 0|(1-P)| 0\rangle_{\text {pert }}=\sum_{n=0}^{\infty} c_{n} \alpha_{s}^{n} .
$$

As it is stressed in [117-121], this makes any determination of the QCD condensates in terms of composite operator (9.1) ambiguous.

The phenomena of chromomagnetic gluon condensation [15] initiated series of publications by the ITEP group where they used the gluon condensate to improve their perturbative sum rule equations [71,72]..$^{9}$ Modern determination of the gluon condensate numerical value from hadronic $\tau$ decay data and from the charmonium sum rules can be found in the review article of Ioffe [108]. The best values of condensates, extracted from QCD sum rules from experimental data, are given in Table 1 in [108]. These data do not allow to exclude

\footnotetext{
${ }^{9}$ In 1977 the author gave a theoretical seminar on the chromomagnetic gluon condensation [15] in ITEP. At end of the seminar one of the participants, Victor Novikov, on our way back to the metro station by tram, remarked to the author that the theoretical prediction of the chromomagnetic condensate presented at the seminar [15] can be crucial in improving the naive sum rule equations published earlier in [71] by introducing the chromomagnetic condensate in the form of power corrections. A year later, the proposal was realised in [72].
} 
the zero value for the gluon condensate [106-108]. The separation of perturbative and non-perturbative contributions has some arbitrariness [72], as it was pointed out by Ioffe in [108].

Here I reexamined the phenomena of the YM condensation [15]. It is of the chromomagnetic type and it has a numerical value $\Lambda_{Q C D}^{4}$ which is of the order of few hundred $\mathrm{MeV}^{4}$

$$
\begin{aligned}
\left\langle 2 g^{2} \mathcal{F}\right\rangle_{v a c} & =\left\langle\frac{g^{2}}{2} G_{\mu \nu}^{2}\right\rangle_{v a c}=\left\langle g^{2}\left(\overrightarrow{\mathcal{H}}_{a}^{2}-\overrightarrow{\mathcal{E}}_{a}^{2}\right)\right\rangle_{v a c} \\
& =\mu^{4} \exp \left(-\frac{96 \pi^{2}}{b g^{2}(\mu)}\right)=\Lambda_{Q C D}^{4}>0
\end{aligned}
$$

or in terms of the strong coupling constant

$$
\left\langle\frac{\alpha_{s}}{\pi} G_{\mu \nu}^{2}\right\rangle_{v a c}=\left\langle\frac{g^{2}}{4 \pi^{2}} G_{\mu \nu}^{2}\right\rangle_{v a c}=\frac{\Lambda_{Q C D}^{4}}{2 \pi^{2}} .
$$

Data Availability Statement This manuscript has no associated data or the data will not be deposited. [Authors' comment: The necessary data have been included into the Appendix.]

Open Access This article is licensed under a Creative Commons Attribution 4.0 International License, which permits use, sharing, adaptation, distribution and reproduction in any medium or format, as long as you give appropriate credit to the original author(s) and the source, provide a link to the Creative Commons licence, and indicate if changes were made. The images or other third party material in this article are included in the article's Creative Commons licence, unless indicated otherwise in a credit line to the material. If material is not included in the article's Creative Commons licence and your intended use is not permitted by statutory regulation or exceeds the permitted use, you will need to obtain permission directly from the copyright holder. To view a copy of this licence, visit http://creativecomm ons.org/licenses/by/4.0/.

Funded by SCOAP ${ }^{3}$.

\section{Appendix}

The integrals appearing in the effective Lagrangian (2.13) have the following form:

$$
\begin{aligned}
& \int_{0}^{\infty} \frac{d s}{s^{1-k} \sinh ^{2}(a s)}=\frac{4}{(2 a)^{k}} \Gamma(k) \zeta(k-1), \\
& \int_{0}^{\infty} \frac{\cosh (b s) d s}{s^{1-k} \sinh (a s)} \\
& \quad=\frac{\Gamma(k)}{(2 a)^{k}}\left[\zeta \left(k, \frac{1}{2}\left(1-\frac{b}{a}\right)+\zeta\left(k, \frac{1}{2}\left(1+\frac{b}{a}\right)\right], \quad b \neq a,\right.\right.
\end{aligned}
$$

where $k$ can be considered as a dimensional regularisation parameter and the integrals should be calculated in the limit $k \rightarrow-1$ [12]. As far as $b \neq a$, the second integral does not completely coincide with the one appearing in the effective Lagrangian (2.13) and we have to consider its extension. In order to calculate the integral we shall take $b=a-\epsilon$ and consider the limit $\epsilon \rightarrow 0$ :

$$
\begin{aligned}
& \int_{0}^{\infty} \frac{s^{k-1} \cosh ((a-\epsilon) s) d s}{\sinh (a s)} \\
& \quad=\frac{\Gamma(k)}{(2 a)^{k}}\left[\zeta\left(k, \frac{\epsilon}{2 a}\right)+\zeta\left(k,\left(1-\frac{\epsilon}{2 a}\right)\right] .\right.
\end{aligned}
$$

By the definition the Riemann zeta function $\zeta(k, q)$ is [73]

$\zeta(k, q)=\sum_{n=0}^{\infty} \frac{1}{(n+q)^{k}}=\sum_{n=1}^{\infty} \frac{1}{(n+q)^{k}}+\frac{1}{q^{k}}$

and in the limit $q=\epsilon / 2 a \rightarrow 0$ we shall get

$$
\begin{aligned}
& \lim _{\epsilon \rightarrow 0} \zeta\left(k, \frac{\epsilon}{2 a}\right)=\zeta(k)+\left(\frac{2 a}{\epsilon}\right)^{k}, \\
& \lim _{\epsilon \rightarrow 0} \zeta\left(k,\left(1-\frac{\epsilon}{2 a}\right)=\zeta(k) .\right.
\end{aligned}
$$

Thus the second integral in (10.1) takes the following form:

$$
\begin{aligned}
& \int_{0}^{\infty} \frac{s^{k-1} \cosh ((a-\epsilon) s) d s}{\sinh (a s)}=\frac{\Gamma(k)}{(2 a)^{k}}\left[2 \zeta(k)+\left(\frac{2 a}{\epsilon}\right)^{k}\right] \\
& =\frac{2 \Gamma(k) \zeta(k)}{(2 a)^{k}}+\frac{\Gamma(k)}{\epsilon^{k}}
\end{aligned}
$$

where the last term is field independent and can be subtracted from the effective Lagrangian.

The integrals appearing in Yang-Mills effective Lagrangian (6.1) have the form [12]

$$
\begin{aligned}
& \int_{0}^{\infty} \frac{d s}{s^{1-k} \sinh (a s)}=\frac{2^{k}-1}{2^{k-1} a^{k}} \Gamma(k) \zeta(k), \\
& \int_{0}^{\infty} \frac{\cosh (a s) d s}{s^{1-k} \sinh ^{2}(a s)}=\frac{2^{k-1}-1}{2^{k-2} a^{k}} \Gamma(k) \zeta(k-1) \\
& \int_{0}^{\infty} \frac{\sin (a s) d s}{s^{1-k}}=\frac{\Gamma(k)}{a^{k}} \sin \frac{k \pi}{2}, \\
& \int_{0}^{\infty} \frac{\cos (a s) d s}{s^{1-k}}=\frac{\Gamma(k)}{a^{k}} \cos \frac{k \pi}{2} .
\end{aligned}
$$

The Lagrangian should be calculated in the limit $k \rightarrow-1$.

\section{References}

1. F. Sauter, Uber das Verhalten eines Elektrons im homogenen elektrischen Feld nach der relativistischen Theorie Diracs. Z. Phys. 69, 742 (1931). https://doi.org/10.1007/BF01339461

2. W. Heisenberg, Bemerkungen zur Diracschen Theorie des Positrons. Z. Phys. 90, 209 (1934)

3. H. Euler, B. Kockel, Über die Streuung von Licht an Licht nach der Diracschen Theorie. Naturwiss 23, 246 (1935)

4. W. Heisenberg, H. Euler, Consequences of Dirac's theory of positrons. Z. Phys. 98, 714 (1936)

5. J.S. Schwinger, On gauge invariance and vacuum polarization. Phys. Rev. 82, 664 (1951). https://doi.org/10.1103/PhysRev.82. 664

6. S.R. Coleman, E.J. Weinberg, Radiative corrections as the origin of spontaneous symmetry breaking. Phys. Rev. D 7, 1888 (1973). https://doi.org/10.1103/PhysRevD.7.1888

7. V.S. Vanyashin, M.V. Terentev, The vacuum polarization of a charged vector field. Zh. Eksp. Teor. Fiz. 48(2), 565 (1965) 
8. V.S. Vanyashin, M.V. Terentev, The vacuum polarization of a charged vector field. Sov. Phys. JETP 212, 375 (1965)

9. V.V. Skalozub, The vacuum polarization of the charged vector field in the renormalized theory. Yad. Fiz. 21, 1337 (1975)

10. M.R. Brown, M.J. Duff, Exact results for effective lagrangians. Phys. Rev. D 11, 2124 (1975). https://doi.org/10.1103/PhysRevD. 11.2124

11. M.J. Duff, M. Ramon-Medrano, On the effective Lagrangian for the Yang-Mills field. Phys. Rev. D 12, 3357 (1975). https://doi. org/10.1103/PhysRevD.12.3357

12. G.K. Savvidy, Vacuum polarisation by intensive gauge fields, $\mathrm{PhD}$ (1977). http://www.inp.demokritos.gr/ savvidy/phd.pdf

13. I.A. Batalin, S.G. Matinyan, G.K. Savvidy, Vacuum polarization by a source-free gauge field. Sov. J. Nucl. Phys. 26, 214 (1977)

14. I.A. Batalin, S.G. Matinyan, G.K. Savvidy, Vacuum polarization by a source-free gauge field. Yad. Fiz. 26, 407 (1977)

15. G.K. Savvidy, Infrared instability of the vacuum state of gauge theories and asymptotic freedom. Phys. Lett. B 71, 133 (1977). https://doi.org/10.1016/0370-2693(77)90759-6

16. S.G. Matinyan, G.K. Savvidy, Vacuum polarization induced by the intense gauge field. Nucl. Phys. B 134, 539 (1978). https:// doi.org/10.1016/0550-3213(78)90463-7

17. Y.B. Zel'dovich, The cosmological constant and the theory of elementary particles. Sov. Phys. Usp. 11, 381 (1968)

18. Y.B. Zel'dovich, The cosmological constant and the theory of elementary particles. Usp. Fiz. Nauk 95, 209 (1968). https://doi. org/10.1070/PU1968v011n03ABEH003927

19. Y.B. Zel'dovich, The cosmological constant and the theory of elementary particles. JETP Lett. 6, 316 (1967)

20. S. Weinberg, The cosmological constant problem. Rev. Mod. Phys. 61, 1-23 (1989)

21. A.D. Linde, Is the cosmological constant really a constant? JETP Lett. 19, 183 (1974)

22. A.D. Linde, Is the cosmological constant really a constant? Pisma Zh. Eksp. Teor. Fiz. 19, 320 (1974)

23. A.D. Linde, Infrared problem in thermodynamics of the YangMills gas. Phys. Lett. B 96, 289 (1980). https://doi.org/10.1016/ 0370-2693(80)90769-8

24. A.G. Riess et al. [Supernova Search Team], Observational evidence from supernovae for an accelerating universe and a cosmological constant, Astron. J. 116, 1009 (1998). https://doi.org/10. 1086/300499. arXiv:astro-ph/9805201

25. J.L. Tonry et al. [Supernova Search Team], Cosmological results from high-z supernovae. Astrophys. J. 594, 1 (2003). https://doi. org/10.1086/376865. arXiv:astro-ph/0305008

26. S. Perlmutter et al. [Supernova Cosmology Project Collaboration], Measurements of Omega and Lambda from 42 high redshift supernovae. Astrophys. J. 517, 565 (1999). https://doi.org/ 10.1086/307221. arXiv:astro-ph/9812133

27. M. Betoule et al. [SDSS Collaboration], Improved cosmological constraints from a joint analysis of the SDSS-II and SNLS supernova samples, Astron. Astrophys. 568, A22 (2014). https://doi. org/10.1051/0004-6361/201423413. arXiv:1401.4064 [astroph.CO]

28. R. Adam et al. [Planck Collaboration], Planck 2015 results. I. Overview of products and scientific results. Astron. Astrophys. 594, A1 (2016). https://doi.org/10.1051/0004-6361/201527101. arXiv:1502.01582 [astro-ph.CO]

29. N. Aghanim et al. [Planck Collaboration], Planck 2018 results. VI. Cosmological parameters. arXiv:1807.06209 [astro-ph.CO]

30. H.B. Nielsen, P. Olesen, Vortex line models for dual strings. Nucl. Phys. B 61, 45 (1973). https://doi.org/10.1016/ 0550-3213(73)90350-7

31. N.K. Nielsen, P. Olesen, Electric vortex lines from the YangMills theory. Phys. Lett. B 79, 304 (1978). https://doi.org/10. 1016/0370-2693(78)90249-6
32. C.A. Flory, Stability properties of an abelianized chromoelectric flux tube. Phys. Rev. D 29, 722 (1984). https://doi.org/10.1103/ PhysRevD.29.722

33. M. Gyulassy, A. Iwazaki, Quark and gluon pair production in Su(n) covariant constant fields. Phys. Lett. B 165, 157 (1985). https://doi.org/10.1016/0370-2693(85)90711-7

34. M. Gyulassy, H.T. Elze, A. Iwazaki, D. Vasak, Introduction to quantum chromo transport theory for quark - gluon plasmas, in Changchun 1986, Proceedings, nuclear phase transitions and heavy ion reactions, 83-112. Lawrence Berkeley Lab. - LBL22072 (86,REC.NOV.)

35. N.K. Nielsen, P. Olesen, An unstable Yang-Mills field mode. Nucl. Phys. B 144, 376 (1978). https://doi.org/10.1016/ 0550-3213(78)90377-2

36. V.V. Skalozub, On restoration of spontaneously broken symmetry in magnetic field. Yad. Fiz. 28, 228 (1978)

37. H.B. Nielsen, Approximate QCD lower bound for the bag constant B. Phys. Lett. B 80, 133 (1978). https://doi.org/10.1016/ 0370-2693(78)90326-X

38. J. Ambjorn, N.K. Nielsen, P. Olesen, A hidden Higgs Lagrangian in QCD. Nucl. Phys. B 152, 75 (1979). https://doi.org/10.1016/ 0550-3213(79)90080-4

39. H.B. Nielsen, M. Ninomiya, A bound on bag constant and Nielsen-Olesen unstable mode in QCD. Nucl. Phys. B 156, 1 (1979). https://doi.org/10.1016/0550-3213(79)90490-5

40. H.B. Nielsen, P. Olesen, A quantum liquid model for the QCD vacuum: gauge and rotational invariance of domained and quantized homogeneous color fields. Nucl. Phys. B 160, 380 (1979). https://doi.org/10.1016/0550-3213(79)90065-8

41. H.B. Nielsen, M. Ninomiya, Instanton correction to some vacuum energy densities and the bag constant. Nucl. Phys. B 163, 57 (1980). https://doi.org/10.1016/0550-3213(80)90390-9

42. H.B. Nielsen, P. Olesen, Quark confinement in a random color magnetic ether, NBI-HE-79-45

43. J. Ambjorn, P. Olesen, On the formation of a random color magnetic quantum liquid in QCD. Nucl. Phys. B 170, 60 (1980). https://doi.org/10.1016/0550-3213(80)90476-9

44. J. Ambjorn, P. Olesen, A color magnetic vortex condensate in QCD. Nucl. Phys. B 170, 265 (1980). https://doi.org/10.1016/ 0550-3213(80)90150-9

45. V.V. Skalozub, Nonabelian gauge theories in external electromagnetic field (in Russian). Yad. Fiz. 31, 798 (1980)

46. H. Leutwyler, Vacuum fluctuations surrounding soft gluon fields. Phys. Lett. B 96, 154 (1980). https://doi.org/10.1016/ 0370-2693(80)90234-8

47. H. Leutwyler, Constant gauge fields and their quantum fluctuations. Nucl. Phys. B 179, 129 (1981). https://doi.org/10.1016/ 0550-3213(81)90252-2

48. C.A. Flory, A selfdual gauge field, its quantum fluctuations, and interacting fermions. Phys. Rev. D 28, 1425 (1983). https://doi. org/10.1103/PhysRevD.28.1425

49. D. Kay, R. Parthasarathy, K.S. Viswanathan, Constant self-dual Abelian gauge fields and fermions in SU(2) gauge theory. Phys. Phys. D 28, 3116-3120 (1983)

50. W. Dittrich, M. Reuter, Effective QCD Lagrangian with zeta function regularization. Phys. Lett. B 128, 321 (1983). https://doi.org/ 10.1016/0370-2693(83)90268-X

51. D. Zwanziger, Nonperturbative modification of the FaddeevPopov formula and banishment of the naive vacuum. Nucl. Phys. B 209, 336 (1982). https://doi.org/10.1016/0550-3213(82)90260-7

52. C.A. Flory, Covariant constant chromomagnetic fields and elimination of the one loop instabilities, preprint, SLAC-PUB-3244. http://www-public.slac.stanford.edu/sciDoc/docMeta.aspx? slacPubNumber=SLAC-PUB-3244; https://lib-extopc.kek.jp/ preprints/PDF/1983/8312/8312331.pdf 
53. D. Kay, Unstable modes, zero modes, and phase transitions in QCD, Ph.D Thesis. Simon Fraser University (1985)

54. D. Kay, A. Kumar, R. Parthasarathy, Vacuum in SU(2) Yang-Mills theory. Mod. Phys. Lett. A 20, 1655-1662 (2005)

55. Y.M. Cho, M.L. Walker, Stability of monopole condensation in SU(2) QCD. Mod. Phys. Lett. A 19, 2707 (2004). https://doi.org/ $10.1142 / \mathrm{S} 0217732304015750$

56. S.L. Adler, J.C. Collins, A. Duncan, Energy-momentum-tensor trace anomaly in spin 1/2 QED. Phys. Rev. D 15, 1712 (1977)

57. J.C. Collins, A. Duncan, S.D. Joglekar, Trace and dilatation anomalies in gauge theories. Phys. Rev. D 16, 438 (1977)

58. N.K. Nielsen, The energy-momentum tensor in a non-Abelian quark gluon theory. Nucl. Phys. B 120, 212 (1977)

59. P. Minkowski, On the anomalous divergence of the dilatation current in gauge theories, Bern preprint (1976)

60. M.J. Duff, Observations on conformal anomalies. Nucl. Phys. B 125, 334 (1977). https://doi.org/10.1016/0550-3213(77)90410-2

61. S.L. Adler, Einstein gravity as a symmetry-breaking effect in quantum field theory. Rev. Mod. Phys. 54, 729 (1982) [Erratum: Rev. Mod. Phys. 55, 837 (1983)]. https://doi.org/10.1103/ RevModPhys.54.729

62. G. Savvidy, Generalisation of the Yang-Mills theory. Int. J. Mod. Phys. A 31, 1630003 (2016). https://doi.org/10.1142/ S0217751X16300039; Proceedings of the conference on 60 years of Yang-Mills gauge field theories. Nanyang Technological University, Singapore, 25-28 May 2015. https://doi.org/10.1142/ 9789814725569-0015

63. A.I. Milshtein, Y.F. Pinelis, Properties of the photon polarisation operator in a long wave vacuum field in QCD. Phys. Lett. B 137, 235 (1984). https://doi.org/10.1016/0370-2693(84)90236-3

64. G. Baseyan, S. Matinyan, G. Savvidy, Nonlinear plane waves in the massless Yang-Mills theory. Pisma Zh. Eksp. Teor. Fiz. 29, 641-644 (1979)

65. S. Matinyan, G. Savvidy, N. Ter-Arutyunyan-Savvidi, Classical Yang-Mills mechanics. Nonlinear colour oscillations. Zh. Eksp. Teor. Fiz. 80, 830-838 (1980)

66. G. Savvidy, The Yang-Mills classical mechanics as a Kolmogorov system. Phys. Lett. B 130, 303-307 (1983)

67. G. Savvidy, The Yang-Mills quantum mechanics. Phys. Lett. B 159, 325-329 (1985)

68. G. Savvidy, Classical and quantum mechanics of non-Abelian gauge fields. Nucl. Phys. 246, 302-334 (1984)

69. T. Banks, W. Fischler, S.H. Shenker, L. Susskind, M theory as a matrix model: a conjecture. Phys. Rev. D 55, 5112 (1997)

70. T. Anous, C. Cogburn, Mini-BFSS matrix model in silico. Phys. Rev. D 100, 066023 (2019)

71. V.A. Novikov, L.B. Okun, M.A. Shifman, A.I. Vainshtein, M.B. Voloshin, V.I. Zakharov, Sum rules for charmonium and charmed mesons decay rates in quantum chromodynamics. Phys. Rev. Lett. 38, 626 (1977) [Erratum: Phys. Rev. Lett. 38, 791 (1977)]. https:// doi.org/10.1103/PhysRevLett.38.791.2. https://doi.org/10.1103/ PhysRevLett.38.626

72. V.I. Zakharov, Gluon condensate and beyond. Int. J. Mod. Phys. A 14, 4865 (1999). https://doi.org/10.1142/S0217751X9900230X. arXiv:hep-ph/9906264

73. I.S. Gradshteyn, I.M. Ryzhik, Table of Integrals, Series and Products (Academic Press, New York, 1972)

74. R. Pasechnik, G. Prokhorov, O. Teryaev, Mirror QCD and cosmological constant. Universe 3(2), 43 (2017). https://doi.org/10. 3390/universe3020043. arXiv:1609.09249 [hep-ph]

75. A. Addazi, A. Marcianò, R. Pasechnik, G. Prokhorov, Mirror symmetry of quantum Yang-Mills vacua and cosmological implications. Eur. Phys. J. C 79(3), 251 (2019). https://doi.org/10.1140/ epjc/s10052-019-6780-x
76. J.H. Oort, The force exerted by the stellar system in the direction perpendicular to the galactic plane and some related problems. Bull. Astron. Inst. Netherlands 6, 249 (1932)

77. F. Zwicky, On the masses of nebulae and of clusters of nebulae. Astrophys. J. 86, 217 (1937)

78. K. Nishijima, M.H. Saffouri, CP invariance and the shadow universe. Phys. Rev. Lett. 14, 205 (1965)

79. R. Foot, Experimental implications of mirror matter-type dark matter. Int. J. Mod. Phys. A 19, 3807 (2004). https://doi.org/10. 1142/S0217751X04020087. arXiv:astro-ph/0309330

80. Z. Berezhiani, Mirror world and its cosmological consequences. Int. J. Mod. Phys. A 19, 3775 (2004). https://doi.org/10.1142/ S0217751X04020075. arXiv:hep-ph/0312335

81. R. Barbieri, T. Gregoire, L.J. Hall, Mirror world at the large hadron collider. arXiv:hep-ph/0509242

82. G.V. Dunne, Heisenberg-Euler effective Lagrangians: basics and extensions. arXiv:hep-th/0406216

83. T. He, P. Mitra, A.P. Porfyriadis, A. Strominger, New symmetries of massless QED. JHEP 1410, 112 (2014). https://doi.org/ 10.1007/JHEP10(2014)112. arXiv:1407.3789 [hep-th]

84. G.L. Pimentel, A.M. Polyakov, G.M. Tarnopolsky, Vacua on the brink of decay. Rev. Math. Phys. 30(07), 1840013 (2018). https:// doi.org/10.1142/S0129055X18400135. arXiv:1803.09168 [hepth]

85. H. Gies, G. Torgrimsson, Critical Schwinger pair production IIuniversality in the deeply critical regime. Phys. Rev. D 95, 016001 (2017). arXiv:1612.00635 [hep-th]

86. W. Pauli, Über Gasentartung und Paramagnetizmus. Zs. Phys. 41, $81(1927)$

87. L. Landau, Diamagnetism of metals. Zs. Phys. 64, 629 (1930)

88. K. Enqvist, P. Olesen, Ferromagnetic vacuum and galactic magnetic fields. Phys. Lett. B 329, 195 (1994). https://doi.org/10. 1016/0370-2693(94)90760-9. arXiv:hep-ph/9402295

89. H. Pagels, E. Tomboulis, Vacuum of the quantum Yang-Mills theory and magnetostatics. Nucl. Phys. B 143, 485 (1978). https:// doi.org/10.1016/0550-3213(78)90065-2

90. L.D. Landau, A.A. Abeikosov, I.M. Halatnikov, Asymptotic expression for the photon green function in quantum electrodynamics. Dokl. Akad. Nauk SSSR 95, 1177 (1954)

91. B.L. Ioffe, Bez retushi, [Without Retouching] (in Russian), Phasis Printing House "Nauka" Moscow, 17-19 (2004)

92. S. Mandelstam, Approximation scheme for QCD. Phys. Rev. D 20, 3223 (1979). https://doi.org/10.1103/PhysRevD.20.3223

93. S. Mandelstam, Review of recent results on QCD and confinement, UCB-PTH-79-9

94. S. Mandelstam, General introduction to confinement. Phys. Rep. 67, 109 (1980). https://doi.org/10.1016/0370-1573(80)90083-6

95. Y. Nambu, Effective abelian gauge fields. Phys. Lett. B 102, 149 (1981). https://doi.org/10.1016/0370-2693(81)91051-0

96. S.L. Adler, T. Piran, Relaxation methods for gauge field equilibrium equations. Rev. Mod. Phys. 56, 1 (1984). https://doi.org/10. 1103/RevModPhys.56.1

97. A. Yildiz, P.H. Cox, Vacuum behavior in quantum chromodynamics. Phys. Rev. D 21, 1095 (1980). https://doi.org/10.1103/ PhysRevD.21.1095

98. J.M. Cornwall, Dynamical mass generation in continuum QCD. Phys. Rev. D 26, 1453 (1982). https://doi.org/10.1103/PhysRevD. 26.1453

99. J.M. Cornwall, A. Soni, Couplings of low lying glueballs to light quarks, gluons, and hadrons. Phys. Rev. D 29, 1424 (1984). https:// doi.org/10.1103/PhysRevD.29.1424

100. L.D. Faddeev, A.J. Niemi, Aspects of electric magnetic duality in SU(2) Yang-Mills theory. Phys. Lett. B 525, 195 (2002). https://doi.org/10.1016/S0370-2693(01)01432-0. arXiv:hep-th/0101078 
101. L.D. Faddeev, Notes on divergences and dimensional transmutation in Yang-Mills theory. Theor. Math. Phys. 148, 986 (2006). https://doi.org/10.1007/s11232-006-0095-4. [Teor. Mat. Fiz. 148 (2006) 133]

102. Y.M. Cho, Monopole condensation and mass gap in SU(3) QCD. Int. J. Mod. Phys. A 29, 1450013 (2014). https://doi.org/10.1142/ S0217751X14500134

103. M. Consoli, G. Preparata, On the stability of the perturbative ground state in nonabelian Yang-Mills theories. Phys. Lett. B 154, 411 (1985). https://doi.org/10.1016/0370-2693(85)90420-4

104. T.R. Taylor, G. Veneziano, Strings and $D=4$. Phys. Lett. B 212, 147 (1988). https://doi.org/10.1016/0370-2693(88)90515-1

105. E.V. Shuryak, Theory and phenomenology of the QCD vacuum. Phys. Rep. 115, 151 (1984). https://doi.org/10.1016/ 0370-1573(84)90037-1

106. K. Zyablyuk, Gluon condensate and c quark mass in pseudoscalar sum rules at three loop order. JHEP 0301, 081 (2003). https://doi. org/10.1088/1126-6708/2003/01/081. arXiv:hep-ph/0210103

107. A. Samsonov, Gluon condensate in charmonium sum rules for the axial-vector current. arXiv:hep-ph/0407199

108. B.L. Ioffe, V.S. Fadin, L.N. Lipatov, Quantum chromodynamics: perturbative and nonperturbative aspects. Camb. Monogr. Part. Phys. Nucl. Phys. Cosmol. 30 (2010). https://doi.org/10.1017/ CBO9780511711817

109. M. Reuter, C. Wetterich, Indications for gluon condensation for nonperturbative flow equations. arXiv:hep-th/9411227

110. M. Reuter, C. Wetterich, Search for the QCD ground state. Phys. Lett. B 334, 412 (1994). https://doi.org/10.1016/ 0370-2693(94)90707-2. arXiv:hep-ph/9405300

111. M. Reuter, C. Wetterich, Effective average action for gauge theories and exact evolution equations. Nucl. Phys. B 417, 181 (1994). https://doi.org/10.1016/0550-3213(94)90543-6
112. C. Wetterich, Exact evolution equation for the effective potential. Phys. Lett. B 301, 90 (1993). https://doi.org/10.1016/ 0370-2693(93)90726-X. arXiv:1710.05815 [hep-th]

113. F.J. Dyson, Divergence of perturbation theory in quantum electrodynamics. Phys. Rev. 85, 631 (1952). https://doi.org/10.1103/ PhysRev.85.631

114. L.N. Lipatov, Divergence of the perturbation theory series and the quasiclassical theory. Sov. Phys. JETP 45, 216 (1977)

115. L.N. Lipatov, Divergence of the perturbation theory series and the quasiclassical theory. Zh. Eksp. Teor. Fiz. 72, 411 (1977)

116. G. 't Hooft, in Proceedings of the International School of Subnuclear Physics: The Whys of Subnuclear Physics, Erice, 1977, ed. by A. Zichichi (Plenum, New York, 1979)

117. A. Di Giacomo, G.C. Rossi, Extracting the vacuum expectation value of the quantity $\alpha \times G^{2} / \pi$ from gauge theories on a lattice. Phys. Lett. B 100, 481 (1981). https://doi.org/10.1016/ 0370-2693(81)90609-2

118. J. Kripfganz, Gluon condensate From SU(2) lattice gauge theory. Phys. Lett. B 101, 169 (1981). https://doi.org/10.1016/ 0370-2693(81)90666-3

119. A. Di Giacomo, G. Paffuti, Precise determination of vacuum expectation value of $\alpha \times G^{2} / \pi$ from lattice gauge theories. Phys. Lett. B 108, 327 (1982). https://doi.org/10.1016/ 0370-2693(82)91204-7

120. E.M. Ilgenfritz, M. Muller-Preussker, SU(3) gluon condensate from lattice MC data. Phys. Lett. B 119, 395 (1982). https://doi. org/10.1016/0370-2693(82)90698-0

121. G.S. Bali, C. Bauer, A. Pineda, Model-independent determination of the gluon condensate in four-dimensional SU(3) gauge theory. Phys. Rev. Lett. 113, 092001 (2014). https://doi.org/10. 1103/PhysRevLett.113.092001. arXiv:1403.6477 [hep-ph] 